\title{
Measurement of the shadowing of high-energy cosmic rays by the Moon: A search for TeV-energy antiprotons
}

\section{The L3 Collaboration}

P. Achard ${ }^{\mathrm{a}}$, O. Adriani ${ }^{\mathrm{b}}$, M. Aguilar-Benitez ${ }^{\mathrm{c}}$, M. van den Akker ${ }^{\mathrm{d} J}$. Alcaraz ${ }^{\mathrm{c}}$, G. Alemanni ${ }^{\text {e }}$, J. Allaby ${ }^{\mathrm{f}}$, A. Aloisio ${ }^{\mathrm{g}}$, M.G. Alviggi g, H. Anderhub ${ }^{\mathrm{h}}$, V.P. Andreev ${ }^{\mathrm{i}, \mathrm{j}}$, F. Anselmo ${ }^{\mathrm{k}}$, A. Arefiev ${ }^{1}$, T. Azemoon ${ }^{\mathrm{m}}$, T. Aziz ${ }^{\mathrm{n}}$, P. Bagnaia ${ }^{\mathrm{o}}$, A. Bajo ${ }^{\mathrm{c}}$, G. Baksay ${ }^{\mathrm{p}}$, L. Baksay ${ }^{\mathrm{p}}$, J. Bähr ${ }^{\mathrm{q}}$, S.V. Baldew ${ }^{\mathrm{r}}$, S. Banerjee ${ }^{\mathrm{n}}$, Sw. Banerjee ${ }^{\mathrm{s}}$, A. Barczyk ${ }^{\mathrm{h}, \mathrm{t}}$, R. Barillère ${ }^{\mathrm{f}}$, P. Bartalini $^{\mathrm{e}}$, M. Basile ${ }^{\mathrm{k}}$, N. Batalova ${ }^{\mathrm{u}}$, R. Battiston ${ }^{\mathrm{v}}, \mathrm{A}$. Bay ${ }^{\mathrm{e}}, \mathrm{F}_{\text {. Becattini }}{ }^{\mathrm{b}}$, U. Becker ${ }^{\mathrm{w}}$, F. Behner ${ }^{\text {h, }}$ L. Bellucci ${ }^{\text {b }}$, R. Berbeco ${ }^{\mathrm{m}}$, J. Berdugo ${ }^{\mathrm{c}}$, P. Berges ${ }^{\mathrm{w}}$, B. Bertucci ${ }^{\mathrm{v}}$, B.L. Betev ${ }^{\mathrm{h}}$, M. Biasini v ${ }^{\text {, M. Biglietti }}{ }^{\mathrm{g}}$, A. Biland ${ }^{\mathrm{h}}$, J.J. Blaising ${ }^{\mathrm{s}}$, S.C. Blyth ${ }^{\mathrm{x}}$, G.J. Bobbink ${ }^{\mathrm{r}}$, A. Böhm ${ }^{\mathrm{y}}$, L. Boldizsar ${ }^{\mathrm{z}}$, B. Borgia ${ }^{\mathrm{o}}$, S. Bottai ${ }^{\mathrm{b}}$, D. Bourilkov $^{\text {h, M. Bourquin }}{ }^{\text {a }, ~ S . ~ B r a c c i n i ~}{ }^{\text {a }}$, J.G. Branson ${ }^{\text {aa }}$, F. Brochu ${ }^{\text {s }}$, J.D. Burger ${ }^{\text {w }}$, W.J. Burger ${ }^{\mathrm{v}}$, X.D. Cai ${ }^{\mathrm{w}}$, M. Capell ${ }^{\mathrm{w}}$, G. Cara Romeo ${ }^{\mathrm{k}}$, G. Carlino ${ }^{\mathrm{g}}$, A. Cartacci ${ }^{\mathrm{b}}$, J. Casaus ${ }^{\mathrm{c}}$, F. Cavallari ${ }^{\mathrm{o}}$, N. Cavallo ${ }^{\mathrm{ab}}$, C. Cecchi $^{\mathrm{v}}$, M. Cerrada ${ }^{\text {c }}$, M. Chamizo ${ }^{\text {a }}$, T. Chiarusi ${ }^{b}$, Y.H. Chang ${ }^{\text {ac }}$, M. Chemarin ${ }^{\text {ad }}$, A. Chen ${ }^{\text {ac }}$, G. Chen ${ }^{\text {ae }}$, G.M. Chen ${ }^{\text {ae }}$, H.F. Chen ${ }^{\text {af }}$, H.S. Chen ${ }^{\text {ae }}$, G. Chiefari ${ }^{g}$, L. Cifarelli ${ }^{\mathrm{ag}}, \mathrm{F}$. Cindolo ${ }^{\mathrm{k}}$, I. Clare ${ }^{\mathrm{w}}, \mathrm{R}$. Clare ${ }^{\text {ah }}, \mathrm{G}$. Coignet ${ }^{\mathrm{s}}, \mathrm{N}$. Colino ${ }^{\mathrm{c}}$, S. Costantini ${ }^{\circ}$, B. de la Cruz ${ }^{c}, \mathrm{~S}$. Cucciarelli v, R. de Asmundis ${ }^{\mathrm{g}}$, P. Déglon ${ }^{\mathrm{a}}$, J. Debreczeni $^{z}$, A. Degré ${ }^{\mathrm{s}}, \mathrm{K}$. Dehmelt ${ }^{\mathrm{p}}$, K. Deiters ${ }^{\mathrm{t}}$, D. della Volpe ${ }^{\mathrm{g}}$, E. Delmeire ${ }^{\text {a }}$, P. Denes ${ }^{\text {ai }}$, F. DeNotaristefani ${ }^{\circ}$, A. De Salvo ${ }^{\text {h }}$, M. Diemoz ${ }^{\circ}$, M. Dierckxsens ${ }^{\mathrm{r}}$, L.K. Ding ${ }^{\mathrm{ae}}$, C. Dionisi ${ }^{\circ}$, M. Dittmar ${ }^{\mathrm{h}}$, A. Doria ${ }^{\mathrm{g}}$, M.T. Dova ${ }^{\text {aj, }, 1}$, D. Duchesneau ${ }^{\text {s, }, ~ M . ~ D u d a ~}{ }^{\mathrm{y}}, \mathrm{I}_{\text {. Duran }}{ }^{\mathrm{ak}}$, B. Echenard ${ }^{\mathrm{a}}$, A. Eline ${ }^{\text {f }}$, A. El Hage ${ }^{\text {y }}$, H. El Mamouni ${ }^{\text {ad }}$, A. Engler ${ }^{x}$, F.J. Eppling ${ }^{\text {w }}$, P. Extermann ${ }^{\text {a }}$, G. Faber ${ }^{\text {h, M.A. Falagan }}{ }^{\text {c }}$, S. Falciano ${ }^{\circ}$, A. Favara ${ }^{\text {al, }}$, J. Fay ${ }^{\text {ad }}$, O. Fedin ${ }^{\text {j, M. Felcini }}{ }^{\text {h }}$, T. Ferguson ${ }^{x}$, H. Fesefeldt ${ }^{y}$, E. Fiandrini ${ }^{v}$, J.H. Field $^{\text {a }}$, F. Filthaut ${ }^{\text {d }}$, P.H. Fisher ${ }^{\text {w }}$,W. Fisher ${ }^{\text {ai }}$, I. Fisk ${ }^{\text {aa }}$, G. Forconi ${ }^{\text {w }}$,

\footnotetext{
${ }^{1}$ Also supported by CONICET and Universidad Nacional de La Plata, CC 67, 1900 La Plata, Argentina.
} 
K. Freudenreich h, C. Furetta ${ }^{\text {am }}$, Yu. Galaktionov ${ }^{1, w}$, S.N. Ganguli ${ }^{\text {n }}$, P. Garcia-Abia ${ }^{\mathrm{c}}$, M. Gataullin ${ }^{\text {al }}$, S. Gentile ${ }^{\circ}$, S. Giagu ${ }^{\circ}$, Z.F. Gong ${ }^{\text {af }}$, H.J. Grabosch ${ }^{\mathrm{q}}$, G. Grenier ${ }^{\text {ad }}$, O. Grimm ${ }^{\text {h }}$, H. Groenstege ${ }^{\mathrm{r}}$, M.W. Gruenewald ${ }^{\text {an }}$, M. Guida ${ }^{\text {ag }}$, Y.N. Guo ${ }^{\text {ae }}$, V.K. Gupta ${ }^{\text {ai }}$, A. Gurtu ${ }^{\text {n, }}$ L.J. Gutay ${ }^{\text {u }}$, D. Haas ${ }^{\text {ao }}$, Ch. Haller ${ }^{\text {h}}$, D. Hatzifotiadou ${ }^{\text {k }}$, Y. Hayashi ${ }^{\text {ap }}$, Z.X. He ${ }^{\text {aq }}$, T. Hebbeker ${ }^{y}$, A. Hervé ${ }^{\text {, }}$ J. Hirschfelder ${ }^{\text {x }}$, H. Hofer ${ }^{\text {h }}$, H. Hofer jr. ${ }^{\text {h }}$, M. Hohlmann ${ }^{\text {p }}$, G. Holzner ${ }^{\text {h }}$, S.R. Hou ${ }^{\text {ac }}$, A.X. Huo ${ }^{\text {ae }}$, N. Ito ${ }^{\text {ap }}$, B.N. Jin ${ }^{\text {ae }}$, P. Jindal ${ }^{\text {ar }}$, C.L. Jing ${ }^{\text {ae }}$, L.W. Jones ${ }^{\mathrm{m}}$, P. de Jong ${ }^{\mathrm{r}}$,

I. Josa-Mutuberría $^{\text {c, V. Kantserov }}{ }^{\text {q,2 }}$, M. Kaur ${ }^{\text {ar }}$, S. Kawakami ${ }^{\text {ap }}$, M.N. Kienzle-Focacci a , J.K. Kim ${ }^{\text {as }}$, J. Kirkby ${ }^{\text {f }}$, W. Kittel ${ }^{\text {d }}$, A. Klimentov ${ }^{\text {w,1 }}$, A.C. König ' ${ }^{\text {, E. Kok }}{ }^{\text {r }}$, A. Korn ${ }^{\text {w }}$, M. Kopal ${ }^{\text {u, V. Koutsenko }}{ }^{\text {w, }}$, M. Kräber ${ }^{\text {, }}$, H.H. Kuang ${ }^{\text {ae }}$, R.W. Kraemer ${ }^{\text {x }}$, A. Krüger ${ }^{\text {q }}$, J. Kuijpers ${ }^{\text {d }, ~ A . ~ K u n i n ~}{ }^{\text {w }}$, P. Ladron de Guevara ${ }^{\text {c }}$, I. Laktineh ${ }^{\text {ad }}$, G. Landi ${ }^{\text {b }}$, M. Lebeau ${ }^{\text {f }}$, A. Lebedev ${ }^{\text {w }}$, P. Lebrun ${ }^{\text {ad }}$, P. Lecomte ${ }^{\text {h }}$, P. Lecoq ${ }^{\text {f }}$, P. Le Coultre ${ }^{\text {h,* }}$, J.M. Le Goff ${ }^{f}$, Y. Lei ${ }^{\text {ae }}$, H. Leich ${ }^{q}$, R. Leiste ${ }^{q}$, M. Levtchenko ${ }^{\text {am }}$, P. Levtchenko ${ }^{j}, C_{\text {. Li }}{ }^{\text {af }}, \mathrm{L}$ Li $^{\text {ae }}$, Z.C. Li ${ }^{\text {ae }}$, S. Likhoded ${ }^{\mathrm{q}}$, C.H. Lin ${ }^{\text {ac }}$, W.T. Lin ${ }^{\text {ac }}$, F.L. Linde ${ }^{\mathrm{r}}$, L. Lista ${ }^{\mathrm{g}}$, Z.A. Liu ${ }^{\text {ae }}$, W. Lohmann ${ }^{\mathrm{q}}$, E. Longo ${ }^{\circ}$, Y.S. Lu ${ }^{\text {ae }}$, C. Luci ${ }^{\circ}$, L. Luminari ${ }^{\circ}$, W. Lustermann ${ }^{\text {h, W.G. Ma }}{ }^{\text {af }}$, X.H. Ma ${ }^{\text {ae }}$, Y.Q. Ma ${ }^{\text {ae }}$, L. Malgeri ${ }^{\text {a }}$,

A. Malinin ${ }^{1}$, C. Maña ${ }^{\text {c }}$, J. Mans ${ }^{\text {ai }}$, J.P. Martin ${ }^{\text {ad }}$, F. Marzano ${ }^{\circ}$, K. Mazumdar ${ }^{\mathrm{n}}$, R.R. McNeil ${ }^{\mathrm{i}}$, S. Mele ${ }^{\mathrm{f}, \mathrm{g}}$, X.W. Meng ${ }^{\text {ae }}$, L. Merola ${ }^{\mathrm{g}}$, M. Meschini ${ }^{\text {b }}$, W.J. Metzger ${ }^{\text {d }}$, A. Mihul ${ }^{\text {at }}$, A. van Mil ${ }^{\text {d }}$, H. Milcent ${ }^{\text {f', }}$, G. Mirabelli ${ }^{\circ}$, J. Mnich ${ }^{\mathrm{y}}$, G.B. Mohanty ${ }^{\mathrm{n}}$, B. Monteleoni ${ }^{\text {b, }}{ }^{,}$, G.S. Muanza ${ }^{\text {ad }}$, A.J.M. Muijs ${ }^{\text {r }}$, B. Musicar ${ }^{\text {aa }}$, M. Musy ${ }^{o}$, S. Nagy ${ }^{\text {au }}$, R. Nahnhauer ${ }^{\mathrm{q}}$, V.A. Naumov ${ }^{\text {b, }}$, S. Natale ${ }^{\text {a }}$, M. Napolitano ${ }^{\text {g }}$, F. Nessi-Tedaldi ${ }^{\text {h, }}$, H. Newman ${ }^{\text {al }}$, A. Nisati ${ }^{\circ}$, T. Novak ${ }^{\text {d }}$, H. Nowak ${ }^{\mathrm{q}}$, R. Ofierzynski ${ }^{\mathrm{h}}$, G. Organtini ${ }^{\circ}$, I. Pal ${ }^{\mathrm{u}}$, C. Palomares ${ }^{\text {c }}$, P. Paolucci ${ }^{\mathrm{g}}$, R. Paramatti ${ }^{\text {, }}$, J.-F. Parriaud ad, G. Passaleva ${ }^{\text {b }}$, S. Patricelli g, T. Paul aj, M. Pauluzzi ${ }^{\text {v }}$, C. Paus ${ }^{\text {w }}$, F. Pauss ${ }^{\text {h }}$, M. Pedace ${ }^{\circ}$, S. Pensotti ${ }^{\text {am }}$, D. Perret-Gallix ${ }^{\text {s, }}$, B. Petersen ${ }^{\mathrm{d}}$, D. Piccolo ${ }^{\mathrm{g}}$, F. Pierella $^{\mathrm{k}}$, M. Pieri ${ }^{\mathrm{b}}$, M. Pioppi ${ }^{\mathrm{v}}$, P.A. Piroué ${ }^{\mathrm{ai}}$, E. Pistolesi ${ }^{\text {am }}$, V. Plyaskin ${ }^{1}$, M. Pohl ${ }^{a}$, V. Pojidaev ${ }^{\text {b }}$, J. Pothier ${ }^{f}$, D. Prokofiev ${ }^{j}$, J. Quartieri ${ }^{\text {ag }}$, C.R. Qing ${ }^{\text {aq }}$, G. Rahal-Callot ${ }^{\text {h }}$, M.A. Rahaman ${ }^{\text {n }}$, P. Raics ${ }^{\text {au }}$, N. Raja ${ }^{\text {n }}$, R. Ramelli ${ }^{\text {h }}$, P.G. Rancoita ${ }^{\text {am }}$, R. Ranieri ${ }^{\text {b }}$, A. Raspereza ${ }^{\text {, }}$, K.C. Ravindran ${ }^{\text {n }}$, P. Razis ${ }^{\text {av }}$, D. Ren ${ }^{\text {h }}$, M. Rescigno ${ }^{\circ}$, S. Reucroft ${ }^{\text {aj }}$,

\footnotetext{
${ }^{2}$ On leave from the Moscow Physical Engineering Institute (MePhl).

${ }^{3}$ On leave from JINR, RU-141980 Dubna, Russia.

Deceased.

* Corresponding author. Tel.: +41 22767 6026; fax: +41 227671520 .

E-mail addresses: pierre.le.coultre@cern.ch, lecoultr@mail.cern.ch (P. Le Coultre).
} 
P. Rewiersma $^{\mathrm{r}, \mathrm{H}}, \mathrm{S}$. Riemann ${ }^{\mathrm{q}}$, K. Riles ${ }^{\mathrm{m}}$, B.P. Roe ${ }^{\mathrm{m}}$, A. Rojkov ${ }^{\mathrm{b}, \mathrm{d}, \mathrm{h}}$, L. Romero ${ }^{\text {c }}$, A. Rosca ${ }^{\mathrm{q}}$, C. Rosemann ${ }^{\mathrm{y}}$, S. Rosier-Lees ${ }^{\text {ac }}$, C. Rosenbleck ${ }^{\mathrm{y}}$, S. Roth ${ }^{\text {y }}$, J.A. Rubio ${ }^{\text {f }}$, G. Ruggiero ${ }^{\text {b }}$, H. Rykaczewski ${ }^{\text {h }}$, R. Saidi ${ }^{\text {aw }}$, A. Sakharov ${ }^{\text {h }}$, S. Saremi i ${ }^{\text {i }}$ S. Sarkar ${ }^{o}$, J. Salicio ${ }^{\text {f }}$, E. Sanchez ${ }^{c}$, C. Schäfer ${ }^{f}$, V. Schegelsky ${ }^{\text {j }}$, V. Schmitt ${ }^{\text {aw }}$, B. Schoeneich ${ }^{\mathrm{q}}$, H. Schopper ${ }^{\text {ax }}$, D.J. Schotanus ${ }^{\mathrm{d}}$, C. Sciacca ${ }^{\text {g }}$, L. Servoli v, C.Q. Shen ${ }^{\text {ae }}$, S. Shevchenko ${ }^{\text {al }}$, N. Shivarov ${ }^{\text {ay }}$, V. Shoutko ${ }^{\text {w }}$, E. Shumilov ${ }^{1}$, A. Shvorob ${ }^{\text {al }}$, D. Son ${ }^{\text {as }}$, C. Souga ${ }^{\text {ad }}$, P. Spillantini ${ }^{\text {b }}$, M. Steuer ${ }^{\text {w }}$, D.P. Stickland ${ }^{\text {ai }}$, B. Stoyanov ${ }^{\text {ay }}$, A. Straessner ${ }^{\text {a }}$, K. Sudhakar ${ }^{n}$, H. Sulanke ${ }^{\mathrm{q}}$, G. Sultanov ${ }^{\text {ay }}$, L.Z. Sun ${ }^{\text {af }}$, S. Sushkov ${ }^{\mathrm{y}}$, H. Suter ${ }^{\text {h, J.D. Swain }}{ }^{\text {aj }}$, Z. Szillasi ${ }^{\text {p }, 4}$, X.W. Tang ${ }^{\text {ae }}$, P. Tarjan ${ }^{\text {au }}$, L. Tauscher $^{\text {ao }}$, L. Taylor aj, B. Tellili ${ }^{\text {ad }}{ }^{\text {, D. Teyssier }}{ }^{\text {ad }}$, C. Timmermans ${ }^{\text {d }}$, Samuel C.C. Ting ${ }^{\text {w }}$, S.M. Ting ${ }^{\text {w }}$, S.C. Tonwar ${ }^{\mathrm{n}}$, J. Tóth ${ }^{\mathrm{z}}$, G. Trowitzsch ${ }^{\mathrm{q}}$, C. Tully ${ }^{\text {ai }}$, K.L. Tung ${ }^{\text {ae }}$, J. Ulbricht ${ }^{h}$, M. Unger ${ }^{\mathrm{q}}$, E. Valente ${ }^{\mathrm{o}}$, H. Verkooijen ${ }^{\mathrm{r}}$, R.T. Van de Walle ${ }^{\mathrm{d}}$, R. Vasquez ${ }^{\mathrm{u}}$, V. Veszpremi ${ }^{\mathrm{p}}$, G. Vesztergombi ${ }^{\mathrm{z}}$, I. Vetlitsky ${ }^{1}$, D. Vicinanza ${ }^{\text {ag }}$, G. Viertel ${ }^{\text {h }}$, S. Villa ${ }^{\text {ah }}$, M. Vivargent ${ }^{\text {s }}$,

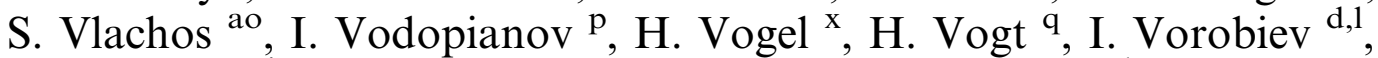
A.A. Vorobyov ${ }^{\mathrm{j}}$, M. Wadhwa ${ }^{\text {ao }}$, R.G. Wang ${ }^{\text {ae }}$, Q. Wang ${ }^{\mathrm{d}}$, X.L. Wang ${ }^{\text {af }}$, X.W. Wang ${ }^{\text {ae }}$, Z.M. Wang ${ }^{\text {af }}$, M. Weber ${ }^{f}$, R. van Wijk ${ }^{r}$, T.A.M. Wijnen ${ }^{\text {d, }}$, H. Wilkens ${ }^{\mathrm{d}}$, S. Wynhoff ${ }^{\text {ai }}$, L. Xia ${ }^{\text {al }}$, Y.P. Xu ${ }^{\text {h }}$, J.S. Xu ${ }^{\text {ae }}$, Z.Z. Xu ${ }^{\text {af }}$, J. Yamamoto ${ }^{\mathrm{m}}$, B.Z. Yang ${ }^{\text {af }}$, C.G. Yang ${ }^{\text {ae }}$, H.J. Yang ${ }^{\mathrm{m}}$, M. Yang ${ }^{\text {ae }}$,

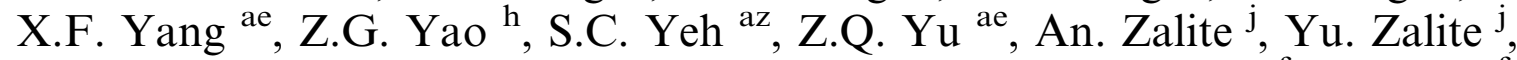
C. Zhang ${ }^{\text {ae }}$, F. Zhang ${ }^{\text {ae }}$, J. Zhang ${ }^{\text {ae }}$, S. Zhang ${ }^{\text {ae }}$, Z.P. Zhang af, J. Zhao af, S.J. Zhou ${ }^{\text {ae }}$, G.Y. Zhu ${ }^{\text {ae }}$, R.Y. Zhu ${ }^{\text {al }}$, Q.Q. Zhu ${ }^{\text {ae }}$, H.L. Zhuang ${ }^{\text {ae }}$,
A. Zichichi ${ }^{\text {f,k,ba }}{ }^{\text {, B. Zimmermann }}{ }^{\text {h }}$, M. Zöller ${ }^{\mathrm{y}}$, A.N.M. Zwart ${ }^{\mathrm{r}}$
${ }^{a}$ University of Geneva, CH-1211 Geneva 4, Switzerland
${ }^{\mathrm{b}}$ University of Florence and INFN, Sezione di Firenze, I-50019, Sesto Fiorentino, Italy
${ }^{\mathrm{c}}$ Centro de Investigaciones Energéticas, Medioambientales y Tecnológicas, CIEMAT, E-28040 Madrid, Spain ${ }^{5}$
d Radboud University and NIKHEF, NL-6525 ED Nijmegen, The Netherlands
${ }^{\mathrm{e}}$ University of Lausanne, CH-1015 Lausanne, Switzerland
${ }^{\mathrm{f}}$ European Laboratory for Particle Physics, CERN, CH-1211 Geneva 23, Switzerland
INFN-Sezione di Napoli and University of Naples, I-80125 Naples, Italy
${ }^{\mathrm{h}}$ Eidgenössische Technische Hochschule, ETH Zürich, CH-8093 Zürich, Switzerland
${ }^{\text {i } L o u i s i a n a ~ S t a t e ~ U n i v e r s i t y, ~ B a t o n ~ R o u g e, ~ L A ~ 70803, ~ U S A ~}$
${ }^{\mathrm{j}}$ Nuclear Physics Institute, St. Petersburg, Russia
${ }^{\mathrm{k}}$ University of Bologna and INFN-Sezione di Bologna, I-40126 Bologna, Italy
${ }^{1}$ Institute of Theoretical and Experimental Physics, ITEP, Moscow, Russia
${ }^{\mathrm{m}}$ University of Michigan, Ann Arbor, MI 48109, USA
${ }^{\mathrm{n}}$ Tata Institute of Fundamental Research, Mumbai (Bombay) 400 005, India

\footnotetext{
${ }^{4}$ Also supported by the Hungarian OTKA fund under contract number T026178.

${ }^{5}$ Supported also by the Comisión Interministerial de Ciencia y Tecnología.
} 
' INFN-Sezione di Roma and University of Rome, "La Sapienza", I-00185 Rome, Italy

${ }^{\mathrm{p}}$ Florida Institute of Technology, Melbourne, FL 32901, USA

' DESY, D-15738 Zeuthen, Germany

${ }^{\mathrm{r}}$ NIKHEF, and University of Amsterdam, NL-1009 DB Amsterdam, The Netherlands

${ }^{\mathrm{s}}$ LAPP,IN2P3-CNRS, BP 110, F-74941 Annecy-le-Vieux Cedex, France

${ }^{\mathrm{t}}$ Paul Scherrer Institut, PSI, CH-5232 Villigen, Switzerland

" Purdue University, West Lafayette, IN 47907, USA

${ }^{\vee}$ INFN-Sezione di Perugia and Università Degli Studi di Perugia, I-06100 Perugia, Italy

${ }^{\mathrm{w}}$ Massachusetts Institute of Technology, Cambridge, MA 02139, USA

${ }^{x}$ Carnegie Mellon University, Pittsburgh, PA 15213, USA

${ }^{y}$ III. Physikalisches Institut, RWTH, D-52056 Aachen, Germany ${ }^{6}$

${ }^{\mathrm{z}}$ Central Research Institute for Physics of the Hungarian Academy of Sciences, H-1525 Budapest 114, Hungary ${ }^{7}$

aa University of California, San Diego, CA 92093, USA

${ }^{\mathrm{ab}}$ INFN-Sezione di Napoli and University of Potenza, I-85100 Potenza, Italy

${ }^{\mathrm{ac}}$ National Central University, Chung-Li, Taiwan, China

${ }^{\text {ad }}$ Institut de Physique Nucléaire de Lyon, IN2P3-CNRS, Université Claude Bernard, F-69622 Villeurbanne, France

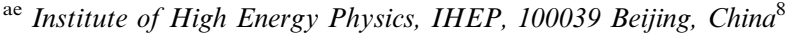

${ }^{\text {af }}$ Chinese University of Science and Technology, USTC, Hefei, Anhui 230 029, China ${ }^{8}$

ag University and INFN, Salerno, I-84100 Salerno, Italy

${ }^{\text {ah }}$ University of California, Riverside, CA 92521, USA

ai Princeton University, Princeton, NJ 08544, USA

${ }^{\text {aj }}$ Northeastern University, Boston, MA 02115, USA

${ }^{\text {ak }}$ University of Santiago de Compostela, E-15706 Santiago, Spain

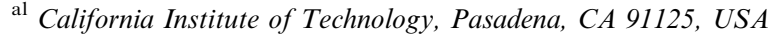

am INFN-Sezione di Milano, I-20133 Milan, Italy

an Department of Experimental Physics, University College Dublin, Belfield, Dublin 4, Ireland

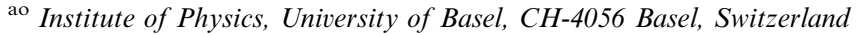

ap ITP, Academia Sinica, 100039 Beijing, China

aq Osaka City University, Osaka 558-8585, Japan

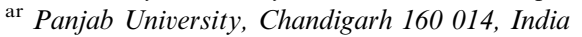

as The Center for High Energy Physics, Kyungpook National University, 702-701 Taegu, Republic of Korea

${ }^{a t}$ Institute of Atomic Physics and University of Bucharest, R-76900 Bucharest, Romania

${ }^{\text {au }}$ KLTE-ATOMKI, H-4010 Debrecen, Hungary ${ }^{9}$

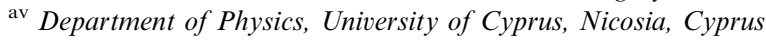

aw Humboldt University, D-10115 Berlin, Germany

ax University of Hamburg, D-22761 Hamburg, Germany

${ }^{\text {ay }}$ Bulgarian Academy of Sciences, Central Laboratory of Mechatronics and Instrumentation, BU-1113 Sofia, Bulgaria

az Department of Physics, National Tsing Hua University, Taiwan, China

ba World Laboratory, FBLJA Project, CH-1211 Geneva 23, Switzerland

Received 8 December 2004; received in revised form 28 January 2005; accepted 3 February 2005

Available online 16 March 2005

\begin{abstract} ations with the L3 + C muon spectrometer at CERN. A significant effect of the Earth magnetic field is observed. Since

\footnotetext{
${ }^{6}$ Supported by the German Bundesministerium für Bildung, Wissenschaft, Forschung und Technologie.

${ }^{7}$ Supported by the Hungarian OTKA fund under contract numbers T019181, F023259 and T037350.

${ }^{8}$ Supported by the National Natural Science Foundation of China.

9 Also supported by the Hungarian OTKA fund under contract number T026178.
}

The shadowing of high-energy cosmic rays by the Moon has been observed with a significance of 9.4 standard devi- 
no event deficit on the east side of the Moon has been observed, an upper limit at $90 \%$ confidence level on the antiproton to proton ratio of 0.11 is obtained for primary energies around $1 \mathrm{TeV}$.

(c) 2005 Elsevier B.V. All rights reserved.

\section{Introduction}

\subsection{Physics motivation}

The effect of the Moon, or the Sun, on cosmic rays was first noted by Clark in 1957 [1]. As these bodies pass overhead they block the particles, so their shadows in the cosmic ray flux must be visible by detectors on Earth.

However the first observation of such a shadowing had to wait for the results of the CYGNUS collaboration in 1991 [2]. There are two reasons for this long delay. First, the particles must be insensitive or weakly sensitive to the Earth magnetic field. Obvious candidates are $\gamma$-rays or energetic cosmic ray particles. The former are very rare and the observation of the latter above the nearly-isotropic large background of low-energy cosmic rays, was only possible with the advent of large extended-air-shower (EAS) detectors, able to collect high statistics. Second, a crucial parameter is the angular resolution of the detectors. The signal over background ratio is inversely proportional to the square of this angular resolution and events are spread out from the expected position due to the finite angular resolution. The performance of the detector has to cope with the angular radius of the Moon (or the Sun), each having approximately a $0.27^{\circ}$ radius, and only at the beginning of the $90 \mathrm{~s}$, the angular resolutions of cosmic ray detectors reached the one-degree level.

Since then, several other experiments, both EAS arrays and large underground detectors have been able to see the Moon-shadow effect [3-7]. The observation is used for a check of the angular resolution of the apparatus and, by comparing the observed position of the deficit to the expected Moon position, to evaluate systematic pointing errors. The understanding of the alignment and of the angular resolution is a key issue for any point-source search.
In 1990, a more challenging use of the Moonshadow effect has been proposed [8]. The use of the Moon collimation, together with the Earth magnetic field, allows a charge determination. Negatively charged primaries are deflected towards the west and positively charged primaries towards the east. If antiprotons are present in the cosmic ray flux, they will generate a shadow on the opposite side of the Moon relative to the shadow from cosmic rays induced from matter. This article discusses a search for cosmic ray antiprotons using the $\mathrm{L} 3+\mathrm{C}$ muon spectrometer of the L3 detector at the CERN LEP accelerator. Published direct measurements of the $\bar{p} / p$ ratio exist only below $40 \mathrm{GeV}$ and this method is sensitive at $\mathrm{TeV}$ energies. Only non-standard sources would be the origin of such high-energy antiprotons. No such study has yet been published using the Earth-Moon system as a spectrometer: EAS arrays (with the exception of the Tibet array) and underground detectors have a too-high detection threshold, so that the effect of the Earth magnetic field is just a small perturbation. This is no longer the case in the $\mathrm{L} 3+\mathrm{C}$ experiment. Due to only $30 \mathrm{~m}$ of overburden, multiple scattering remains small even for low energies and the accumulation rate is much larger than in other underground detectors. Moreover, the measurement of the muon momentum allows for an offline tuning of the threshold, leading to a possible optimisation of the shadow effect. The present study exploits these possibilities and looks at the conditions to set a limit to the $\overline{\mathrm{p}}$ content in the $\mathrm{TeV}$ region.

This section describes the status of cosmic ray antiproton data and summarizes other experimental observations of the Moon shadow by cosmic rays. The experimental setup is presented in Section 2. Section 3 contains a review of the main parameters involved in a Moon-shadow experiment and describes the role of the different Monte Carlo simulations. In particular, the simulation of the 
experimental angular-resolution is checked with the help of two-track events. Data and backgrounds are presented in Section 4. The observation and interpretation of the deficit of events in the Moon direction are described in Section 5. Results concerning the experimental angular-resolution and a possible shadowing effect due to antiprotons are discussed. Conclusions are presented in Section 6.

\subsection{Cosmic-ray antiprotons}

The experimental $\overline{\mathrm{p}} / \mathrm{p}$ ratio below $50 \mathrm{GeV}$ is compatible with a secondary origin of the cosmic ray antiprotons [9]. Data are obtained by balloon-borne experiments and, recently, satellite experiments. The CAPRICE [10] and HEAT [11] collaborations obtained ratios measured between 4 and $50 \mathrm{GeV}$ with a series of balloon flights. The CAPRICE data do not show any flattening of the ratio with increasing energy (ratio $\sim 10^{-3}$ around $40 \mathrm{GeV}$ ) as expected from the secondary production model, in contrast with the last HEAT data point, which sets a limit of $\sim 2 \times 10^{-4}$ above $20 \mathrm{GeV}$. This underlines the limited statistics available up to now in this kind of experiments.

The uncertainties of theoretical models are large below the well defined flux maximum of $2 \mathrm{GeV}$ due to the complexity of production and propagation at low energy. On the contrary, the secondary high-energy flux is predicted with good confidence, all estimates being consistent with each other. Above a few tens of $\mathrm{GeV}$, the antiproton production becomes quite negligible, the flux falling by 3 orders of magnitude below the maximum for antiproton energies around $40 \mathrm{GeV}$. Any experimental hint of antiprotons in these high-energy regions would therefore be of prime importance.

First upper limits [12] on the $\bar{p} / p$ ratio around $1 \mathrm{TeV}$ were presented by the $\mathrm{L} 3+\mathrm{C}$ and the TIBET-AS $\gamma$-collaborations [13] at different conferences. The Tibet array has a worse angular resolution compared to the $\mathrm{L} 3+\mathrm{C}$ experiment and observes a smaller deviation of the Moon shadow due to its sensitivity to higher primary energies.
In reference [14] an upper limit on the $\bar{p} / p$ ratio around $1 \mathrm{TeV}$ is calculated from different measured $\mu^{+} / \mu^{-}$-ratios at ground level with large uncertainties. This indirect determination of a limit is based on cascade calculations which depend on the assumed primary composition and the hadronic interaction cross-sections at high energies [15]. The method has therefore large systematic uncertainties. $\mathrm{L} 3+\mathrm{C}$ has recently measured precisely the muon momentum spectrum, as well as the charge ratio and the angular dependence [16]. Based on to-day's knowledge of the parameters entering the calculation, an estimate of an upper limit on the contribution of antiprotons to the primary flux could not compete with the one presented in this paper.

There are at least three models of exotic sources able to produce high-energy antiprotons: primordial black hole (PBH) evaporation [17], dark-matter neutralino annihilation $[18,19]$ and high-energy antiprotons from extragalactic sources $[14,20]$. In some scenarios, the last two models can provide $\mathrm{a} \overline{\mathrm{p}} / \mathrm{p}$ ratio increase up to the $10 \%$ level in the energy range under investigation. This shows the importance of measuring the antiproton flux at high energy. The antiproton energy-spectrum is of course expected to be different for each type of sources. Putting a limit to the number of antiprotons will, in any case, constrain some of the parameters of the models.

\subsection{Moon-shadow experiments}

EAS arrays were the first detectors to look for a Moon-shadow effect and the first observation was reported by the CYGNUS collaboration [2], with a 4.9 standard deviations (s.d.) significance. The CASA group [3], with a larger array, obtained about the same result. In absence of any dominant point-source, the Moon-shadow experiment provides a unique possibility to make a direct measurement of the angular resolution of the detectors and to verify the pointing accuracy.

Large underground detectors also have the potential to observe the Moon shadow. Backtracking muons to the surface depends on the correct estimation of multiple Coulomb scattering in the large 
rock overburden, a process known to be essentially non-Gaussian. Results have been presented by the MACRO [5,6] and SOUDAN [7] collaborations. These detectors have muon energy-thresholds of several $\mathrm{TeV}$. As a consequence, statistics are low even if the data were accumulated for nearly 10 years. The effect of the geomagnetic field is hardly visible being less important than the observed shift from the origin due to pointing uncertainties. To make the influence of the deflection due to the magnetic field significant, the only possibility is to lower the detection threshold of the primary particles. Locating EAS arrays at very high altitudes is a solution. The TIBET air-shower experiment [4] has been operated since 1990 at $4300 \mathrm{~m}$ above sea level (a.s.1.) and has provided the first unambiguous effect of the geomagnetic field on the Moon shadow.

Imaging-Cherenkov detectors have also been proposed for the observation of the Moon shadow [8]. The search of $\gamma$ sources with this technique was a success, in particular with the observation of the Crab nebula and a handful of other point sources. The application to the Moon-shadow measurement is more difficult as moonlight prohibits the use of visible photons and no Moon shadow was observed with this technique [21].

Another promising technique uses a large volume of water as the detection medium. Photomultiplier tubes detect the Cherenkov radiation produced in the water by relativistic charged particles or photons produced in the primary shower. The MILAGRO collaboration [22,23] built a first prototype, MILAGRITO, running from February 1997 to May 1998, then a full detector, MILAGRO, starting its operation in February 1999. The goal is to be sensitive to primary cosmic rays down to $1 \mathrm{TeV}$ or less, as imaging-Cherenkov detectors, while maintaining an all-sky acceptance and a high-duty cycle like EAS arrays. Preliminary results have shown that the Moon-shadow effect is observed with a significance above 20 s.d. [24]. No result of an antiproton search is yet available.

\section{The $\mathrm{L3}+\mathrm{C}$ detector}

The $\mathrm{L} 3+\mathrm{C}$ detector is part of the $\mathrm{L} 3$ apparatus [25], one of the four particle detectors installed on the LEP Collider. It is located underneath the French-Swiss border at CERN, at 450 ma.s.l. under $30 \mathrm{~m}$ of sedimentary rocks called molasse (density $\sim 7.2 \times 10^{3} \mathrm{~g} / \mathrm{cm}^{2}$ ). It mainly makes use of the muon chamber system which was designed to make a very precise measurement of muons produced in $\mathrm{e}^{+} \mathrm{e}^{-}$collisions. The muon spectrometer consists of two octagonally shaped rings, each with eight "octants", installed in a $12 \mathrm{~m}$ diameter solenoidal magnet which provides a uniform field of 0.5 Tesla along the $\mathrm{e}^{+} \mathrm{e}^{-}$beam direction. Each octant contains precision drift chambers organised in three layers to measure the projection of the muon trajectory onto the plane orthogonal to the magnetic field, and layers of drift cells to measure the projection along the magnetic field direction. Other parts of the L3 detector are not used by L3 + C. To fulfil the specific features of the cosmic ray experiment and to make the running of both L3 and L3 + C completely independent from each other, several systems are specifically added to the L3 setup:

- On top of the magnet, $202 \mathrm{~m}^{2}$ of plastic scintillators are installed to determine the muon arrival time.

- A new trigger and data-acquisition system is built to decouple the L3 and L3 + C operation.

- A precise timing system is devised. It is based on an external GPS module and it includes also $1 \mathrm{~Hz}$ and $10 \mathrm{MHz}$ clocks.

- An air-shower scintillator array is installed on the roof of the surface building to estimate the shower size associated to a detected muon. Its data are not used in the present analysis.

The geometrical acceptance of the detector amounts to about $200 \mathrm{~m}^{2} \mathrm{sr}$ and the muon momentum threshold set by the overburden is $15 \mathrm{GeV}$. The detector was operational in May 1999 and a total of $1.2 \times 10^{10}$ muon triggers were collected up to November 2000, corresponding to an effective live-time of 312 days. Both momentum resolution and detection efficiency are checked using muons from $Z$ decays that $\mathrm{L} 3+\mathrm{C}$ could also detect when the accelerator was running at a centre-of-mass energy equal to the $Z$-boson mass. Studies are extended using muons going through 
two separate octants, both giving a nearly independent measurement of the particle momentum. The momentum resolution is found to be $4.6 \%$ at $45 \mathrm{GeV}$ and $7.4 \%$ at $100 \mathrm{GeV}$. The muon momentum threshold can be adjusted off-line to optimise the results. A detailed description of the $\mathrm{L} 3+\mathrm{C}$ detector and its performances is given in Refs. $[16,26]$.

\section{Experimental considerations and Monte Carlo simulations}

A specific description [27] of the Earth-Moon spectrometer system and of the cosmic ray shadowing effect is implemented in the $\mathrm{L} 3+\mathrm{C}$ simulation with two aims:

- to take into account as accurately as possible the different detector components, the Earth magnetic field, the cosmic ray showering in the atmosphere, the multiple scattering of muons in the molasse, and the reconstruction,

- to understand the relative importance of the various parameters contributing to the measurement of the $\bar{p} / p$ ratio.

The detector properties are described with a Monte Carlo based on the GEANT program [28] which takes into account the effect of energy loss, multiple Coulomb scattering and showering in the detector. The basic version of the model is identical to the L3 simulation package, but specific features required by the $\mathrm{L} 3+\mathrm{C}$ setup, are taken into account, such as the additional scintillators on top of the magnet, or the magnetic field in the coil and yoke of the magnet. For the measurement of cosmic rays originating from the atmosphere, the overburden above the detector must also be included. The energy loss of muons and the smearing of their angular direction is an important issue. The whole surrounding of the L3 detector, consisting mainly of molasse, is introduced, including the access shafts to the experimental cavern and the shielding structures. All main physics processes related to the muon propagation through matter, such as multiple scattering, secondary-particle production including $\delta$-rays, pair production, energy loss and decay are fully simulated.

Special attention is put on the simulation of the muon chambers, by including all inefficiencies due to less efficient and dead cells in the muon detector. The simulated Monte Carlo events are reconstructed and backtracked to the ground level in the same way as the data events.

The simulation of the detector is based on the generation of muons. Instead of performing a full simulation of the air-shower cascade generated by the primary cosmic radiation in the atmosphere, single and double muons are generated above the detector, according to the known angular and energy distributions obtained by a full air shower simulation using the CORSIKA package [29]. The interactions, decays, annihilations and secondary-particle production in the air are fully simulated, according to the current experimental knowledge and to various theoretical models.

\subsection{Angular resolution}

One of the key issues of the Moon-shadow measurement is the experimental angular resolution. The Moon subtends a radius of $0.27^{\circ}$ and the angular resolution has to match this constraint. The following factors are taken into consideration:

- the muon direction with respect to the primary nucleon direction,

- the multiple scattering in the molasse above the detector,

- the intrinsic angular resolution due to the muon chamber resolution, the alignment and the reconstruction precision.

As a result, the angular resolution is a complicated function, depending not only on the muon momentum and on the amount of matter on the particle trajectory, but also on the variables used in the event selection. A good opportunity to characterise the angular precision and check the simulation is given by the study of the space-angle distribution of two-track events in the detector, called "di-muon events" in the following. Muons coming from the decays of mesons originating from the early stages of the shower development 
are produced very high in the atmosphere and move along nearly parallel paths. The angular separation of the two muons is therefore a good measure of the smearing introduced in their direction by all the effects mentioned above. The results of the $\mathrm{L} 3+\mathrm{C}$ simulation is compared with the obtained di-muon data.

\subsubsection{Di-muon analysis}

Di-muon events are selected with cuts requiring a minimum quality of the two tracks. The main goal of the selection is to remove fake di-muon events i.e. events with single muon split into two different tracks because of reconstruction problems. For this purpose, a minimum separation between both tracks is required. Events are further classified into "double-double" "double-single" and "single-single" according to the number of subtracks for each of the reconstructed track, a subtrack being defined for each octant crossed. A muon momentum threshold, defined at the ground surface level, is also imposed on both muons. Except for high muon momenta, large statistics are available and results are mainly dominated by systematic uncertainties. Events simulated with the same sets of cuts are compared with experimental results. Fig. 1 is an example of such a comparison for all events with muon momenta between 50 and $60 \mathrm{GeV}$.

In the following, we define the di-muon angular resolution as $\sigma_{2 \mu}=\mathrm{HWHM} / 1.17$ with $\mathrm{HWHM}$

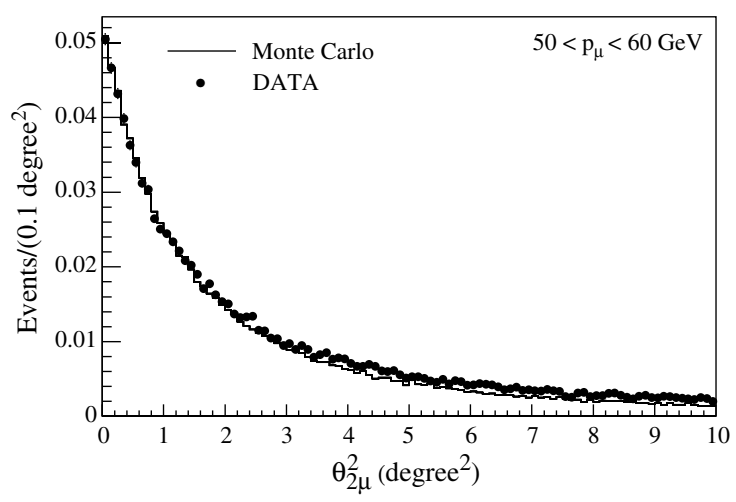

Fig. 1. Distribution of the square of the angle between muons, $\theta_{2 \mu}^{2}$, for data and Monte Carlo. The distributions are normalized to unit area.

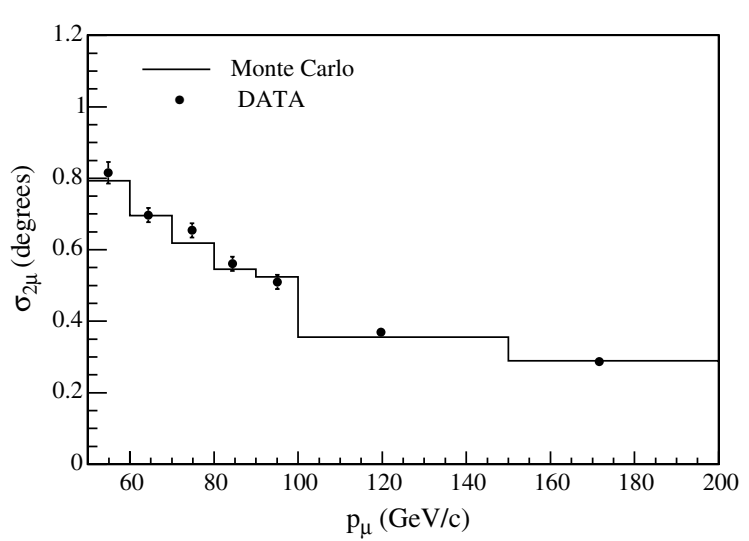

Fig. 2. Di-muon angular resolution versus the muon momentum.

being the half-width at half-maximum of the distribution peak. The observed and expected values of $\sigma_{2 \mu}$ are shown in Fig. 2 as a function of the muon energy for the whole di-muon sample.

Another check of the detector simulation follows from the study of the angular resolution versus the amount of matter crossed by the muons before reaching the detector. The multiple scattering is the main factor in the contributions to the angular resolution from different components. A large range of matter thickness is available by selecting events from the access-shaft direction (minimum energy loss) or from large zenith angles (maximum energy loss).

Experimental results are compared with the detector simulation results in Fig. 3. In all cases, except at large angles, the data is in a rather good agreement with the results from the simulation.

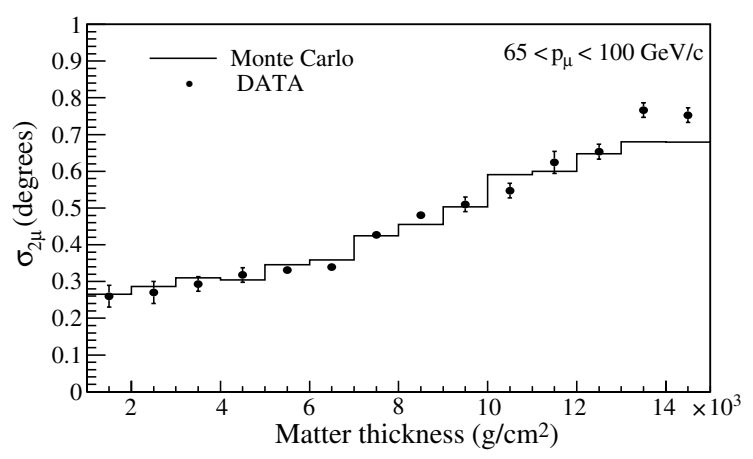

Fig. 3. Di-muon angular resolution versus matter thickness. 


\subsubsection{Angular resolution in the Moon-shadow analysis}

The present analysis uses single-muon events whose angular resolution cannot be deduced from di-muon data. In addition, as the distribution of both type of events inside the air shower are quite different, they do not have the same acceptance. The good agreement observed between data and Monte Carlo in the di-muon analysis gives confidence in the use of the simulation to extract the angular resolution in two ways:

- The angular resolution can be directly extracted from the event deficit in the Moon direction. As already mentioned this is a unique opportunity for an angular resolution measurement and the results will be compared with the simulation.

- Alternatively, the angular resolution can be fully constrained with the help of the simulation of single muon events. The point-spread function for each momentum bin is determined using the same track selection and the same acceptance as for the selected Moon events. The corresponding angular distributions can then be used in the shadow simulation as smearing sources for the angular resolution. Systematic uncertainties are at the level of $5 \%$ or less, better than the angular resolution which can be extracted from the di-muon data. These systematic uncertainties are estimated from the comparison of data and simulation in the dimuon results and from the studies of different production models in the simulation of air showers with the CORSIKA program.

\subsection{Earth magnetic field and calculations of the deflection}

Two models are investigated to describe the geomagnetic field, a simple dipole model and the International Geomagnetic Reference Field model (IGRF) [30]. In the latter, the geomagnetic field is commonly expressed as the gradient of a scalar potential which can be expanded in terms of spherical harmonics. The IGRF consists of a series of values of the coefficients in the expansion based on direct measurements of the geomagnetic field. In fact, the first terms of the expansion can be identified with the field produced by a dipole located at the centre of the Earth. The contributions of the other terms can be considered as perturbations of the main dipole field. Quantitative differences between results of both models have been studied. The main difference is a shift of the southern magnetic pole. As a consequence, differences at the 10\% level are observed at the $\mathrm{L} 3+\mathrm{C}$ location concerning the field intensity. Differences on the magnetic field direction can reach $5^{\circ}$ in the part of the sky where the Moon is visible by the detector. As a result, calculated deflections can differ at the same level. The amount of deflection is overestimated by the dipole model in the largest part of the sky and reaches $10 \%$ for a $1 \mathrm{TeV}$ proton. Consequently only the IGRF model is used in the following.

Two coordinate systems are used. The first is based on the local horizon. The zenith angle $\theta_{z}$ and the azimuth angle $\alpha_{z}$ are determined from the precise position of the detector. The second is an equatorial system using the Earth rotation axis as basis for the two coordinates: declination, $\delta$, and right ascension, RA.

The deflections of the particle trajectories in the magnetic field is described in an additional coordinate system. During its way from the Moon to the Earth, a particle of charge $Z$ and momentum $p$ is subject to the Lorenz force in the field $\vec{B}$ and the deflection $\Delta \theta$ is linked to the particle path $l$, as:

$$
\begin{aligned}
\Delta \theta(\mathrm{mrad})= & 0.3 \cdot \frac{Z}{p(\mathrm{TeV})} \\
& \cdot \mid \int \vec{B}(\text { Tesla }) \times \mathrm{d} \vec{l}(\mathrm{~m}) \mid .
\end{aligned}
$$

Depending on their incident direction at the top of the atmosphere, charged particles traverse different field regions. Thus, for a given particle, the angular deflection is a function of the incident direction, the charge and the momentum of the particle. This can be used to establish a deflection map that gives, for a given momentum, the amount of deflection and its direction. Fig. 4 shows a Moon transit above the sky as seen by the $\mathrm{L} 3+\mathrm{C}$ detector. Each point corresponds to one direction in the sky as computed from the zenith and azimuth angles. During a Moon transit in the sky, the direction of deflection strongly depends on the Moon 


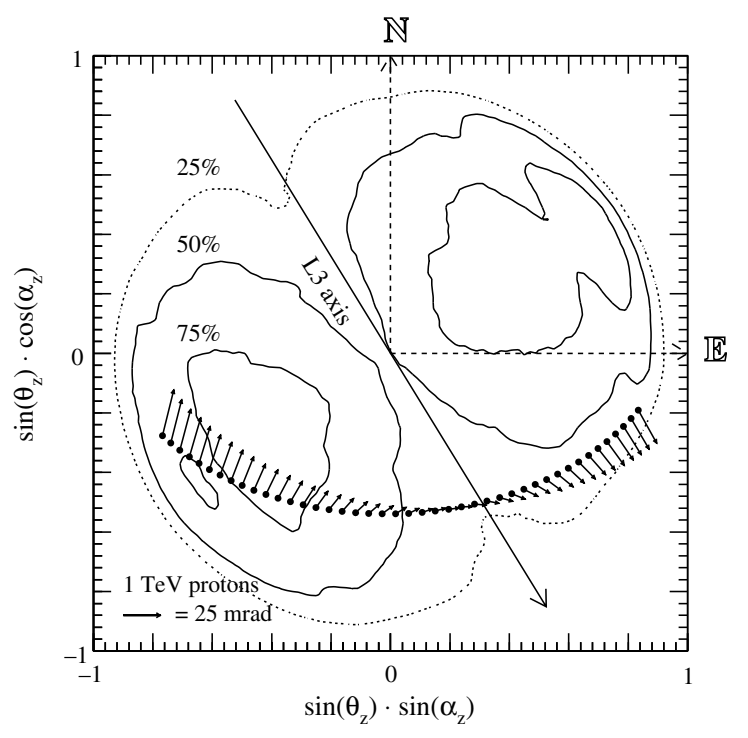

Fig. 4. The Moon passing through the $\mathrm{L} 3+\mathrm{C}$ acceptance. The acceptance is determined by the particular structure of the drift chamber and scintillator assembly. The contour lines correspond to an observed cosmic-ray flux of respectively $75 \%, 50 \%$ and $25 \%$ of the maximum of the flux. A Moon transit is indicated with dots. For each dot, the geomagnetic-deflection direction and amplitude for a $1 \mathrm{TeV}$ proton is indicated by an arrow.

position but the dependence on the momentum is rather small. This leads to the definition of a coordinate system defined for each Moon position in the sky, with coordinates $\theta_{\mathrm{H}}$ and $\theta_{\mathrm{V}}$ respectively parallel and orthogonal to the direction computed for a particle with a given primary momentum (here a $1 \mathrm{TeV}$ proton). The indexes $\mathrm{H}$ and $\mathrm{V}$ stand here respectively for 'horizontal' (parallel deflection) and 'vertical' (perpendicular to deflection). In this way, magnetic deviations will shift the Moon-shadow image along the parallel direction and the shape in the other direction will mainly depend on the angular resolution.

\subsection{Primary cosmic-ray composition}

Energy spectra for various elements, up to a few hundreds $\mathrm{TeV}$ for protons and a few tens $\mathrm{TeV}$ per nucleon $(\mathrm{TeV} / \mathrm{N})$ for heavy elements, have been measured with the use of balloon and satellite experiments. The proton spectra obtained by different experiments are in reasonable agreement.
Results are considerably scattered for other elements, a consequence of limited statistics and uncertainties in the energy calibration.

A compilation of available data [31] proposes the following fit for the flux of particles:

$\Phi=\Phi_{0} E^{-\gamma_{A}}$

with $E$ the energy per nucleus in $\mathrm{TeV}$. The power index for $\mathrm{He}^{4}$ is smaller than the one for protons, and therefore the contribution of $\alpha$ particles increases at high energy. However, recent results from the RUNJOB [32], AMS [33] and BESS [34] collaborations seem to invalidate such behaviour, with a common power index $\sim 2.8$ for proton and helium spectra.

The composition of primary cosmic rays plays an important role for the Moon shadow. It acts remarkably differently for experiments using EAS arrays, Cherenkov or $\mu$ detectors. For the first two methods, the measured signal is proportional to the total energy $E$ of the primary. The third method, characterised by the $\mu$ momentum threshold, is sensitive to the primary energy per nucleon $E_{\mathrm{N}}=E / A$.

Muons with energy $E_{\mu}$ are produced by nucleons of a minimum energy $E_{0}$ with $E_{0} \simeq E_{\mu}$ and thus by nuclei with energy $E>A E_{0}$. In a nucleus, all $A$ nucleons may contribute to the interaction. Let us call $\sigma\left(E_{\mu}, E_{\mathrm{N}}\right)$ the cross section for the production of a muon with energy $E_{\mu}$ by a nucleon with energy $E_{\mathrm{N}}$. If the spectrum has an index $\gamma_{A}$ and the corresponding flux is $\phi_{A}$ at $1 \mathrm{TeV}$, then the number of muons with energy $E_{\mu}$ produced by these nuclei is

$\mathrm{N}_{A}\left(E_{\mu}\right)=A \int_{A E_{0}}^{\infty} \sigma\left(E_{\mu}, E_{\mathrm{N}}\right) \phi_{A} E^{-\gamma_{A}} \mathrm{~d} E$.

If the energy spectrum of all particles follows a power law with the same index $\gamma$ and the probability to yield a muon with energy $E_{\mu}$ does not depend on the energy per nucleon above threshold, the contribution of a nucleus with $A$ nucleons relative to the proton contribution is

$\frac{N_{A}\left(E_{\mu}\right)}{N_{1}\left(E_{\mu}\right)} \propto r_{A} A^{-\gamma+2}$,

where $r_{A}=\frac{\phi_{A}}{\phi_{1}}$ is the relative abundance of a nucleus compared to the proton abundance at 
$1 \mathrm{TeV}$. Using data from Ref. [31], it is found that muons originate at $75.8 \%$ from protons, $17.3 \%$ from helium nuclei and $6.9 \%$ from heavier nuclei.

According to Eq. (1), for a mean primary energy $E_{\text {mean }}$, the mean deflection angle is proportional to the ratio $\frac{Z}{E_{\text {mean }}}$. For experiments sensitive to the total energy, like EAS arrays, the mean primary energy does not depend on $A$. Thus

$$
\langle\Delta \theta\rangle_{\text {EAS }} \propto Z
$$

The position of the Moon-shadow depends only on $Z$, therefore EAS-array experiments expect separate shadows for protons, helium and heavier nuclei.

For a muon experiment, the mean primary energy is proportional to $A E_{0}$. Thus the mean deflection angle is

$\langle\Delta \theta\rangle_{\mu} \propto \frac{Z}{A}$

Muon experiments are sensitive to the ratio $\frac{Z}{A}$, which is equal to 1 for protons, and from 0.5 to 0.4 for heavier nuclei. All shadows from helium and heavier nuclei are almost at the same place. Therefore, in the following, the primary flux for the observed muons will be considered to be $75 \%$ protons and $25 \%$ helium nuclei.

\subsection{Primary cosmic-ray energy spectrum}

There is an energy window for the observation of a magnetic-field effect on the Moon shadow. High primary energies $(\geqslant 10 \mathrm{TeV})$ allow ground level observations with large detectors and relatively good statistics. However the magnetic deflection will be small compared to the angular resolution, making the effect negligible or, at most, appearing just as a small correction. Low primary energies $(\sim 100 \mathrm{GeV})$ are difficult to observe from the ground and large deflections due to the Earth magnetic field will dilute the shadow image and severely limit the sensitivity. L $3+\mathrm{C}$ has a good sensitivity to muons from low-energy primaries. Moreover, the muon energies are measured with good precision. The shadow effect can be observed using different ranges of muon energies, thus selecting samples of different primary energy spectra. For each observed muon energy $E_{\mu}$, a

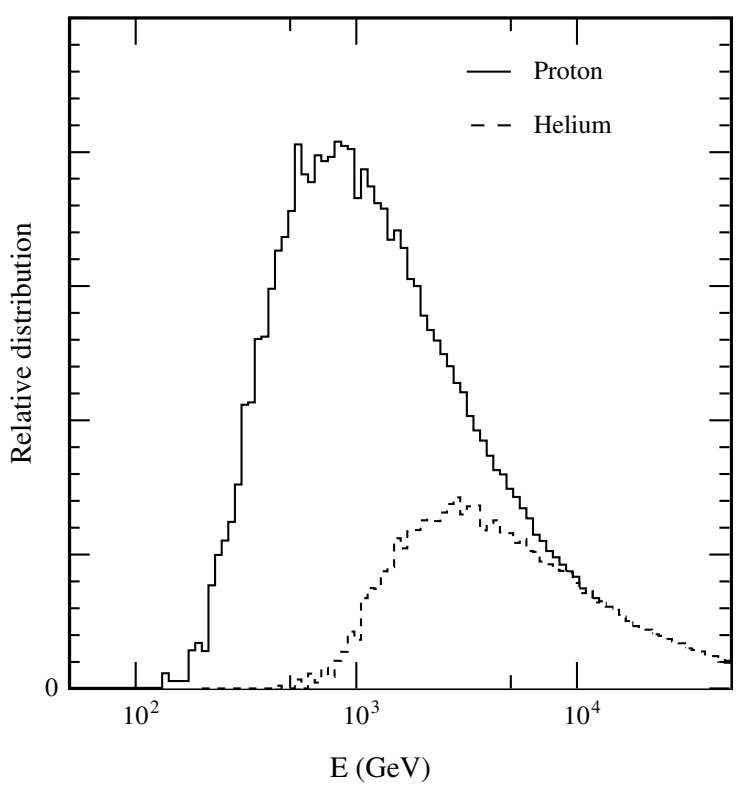

Fig. 5. Distributions of proton and helium primary energies for $E_{\mu}=100 \mathrm{GeV}$. The normalisation between the distributions follows the chosen ratio: $75 \%$ of protons and $25 \%$ of helium. The vertical scale is in arbitrary units.

corresponding primary energy $E$ with $E>E_{\mu}$ is obtained using the shower generation with CORSIKA and the tracking of muons with the detector simulation. Fig. 5 shows the expected proton and helium spectra associated with a detected muon with $E_{\mu}=100 \mathrm{GeV}$. The maximum of the primary energy distribution is around $1 \mathrm{TeV}$ for protons and $4 \mathrm{TeV}$ for helium nuclei.

\subsection{Moon-shadow simulation}

The simulation program tries to reproduce as closely as possible the conditions and the parameters which significantly influence the observation of the Moon shadow. However to make the simulation more efficient, the particles are assumed to be coming from the Moon surface: a positive signal is simulated instead of a deficit. Also, for the same reason, particles are followed backward instead of forward. They are originating from the detector and tracked through the Earth magnetic field up to the Moon. The momentum and identity of the primary particle are extracted from the distributions described above. For the angular 
smearing two methods are applied: either an arbitrary effective angular resolution is used, or the momentum dependent angular information coming from the experimental detector simulation. Events are generated during a time span reproducing the experiment running time and the detector acceptance. Some examples of simulation results are shown in Fig. 6.

The shape of $\theta_{\mathbf{H}}$ becomes more symmetrical as the angular resolution is worsening. In the orthogonal direction, the smearing contribution is mainly coming from the angular resolution only. The expected signals for primary protons and helium nuclei are largely overlapping and no extraction of the helium contribution can be expected from the data. Instead of the $\overline{\mathrm{p}} / \mathrm{p}$ ratio $\left(r_{\overline{\mathrm{p}} / \mathrm{p}}\right)$ mentioned before, the analysis will try to get the " $\overline{\mathrm{p}}$ content" as seen by $\mathrm{L} 3+\mathrm{C}, r=\phi_{\overline{\mathrm{p}}} / \phi_{\text {matter }}$, with $\phi_{\text {matter }}$ the flux responsible of the observed deficit and assuming no anti-Helium contribution. The $\overline{\mathrm{p}} / \mathrm{p}$ ratio itself can then be deduced from this result and the estimated proportion $(75 \%)$ of the deficit due to the proton flux relative to the the total matter flux, as discussed in Section 3.3.

Fig. 7 shows the simulated Moon shadow as it appears for $E_{\mu}>100 \mathrm{GeV}$ in the three coordinate systems: local, equatorial and deflection. Both the offset and the elongation due to the magnetic
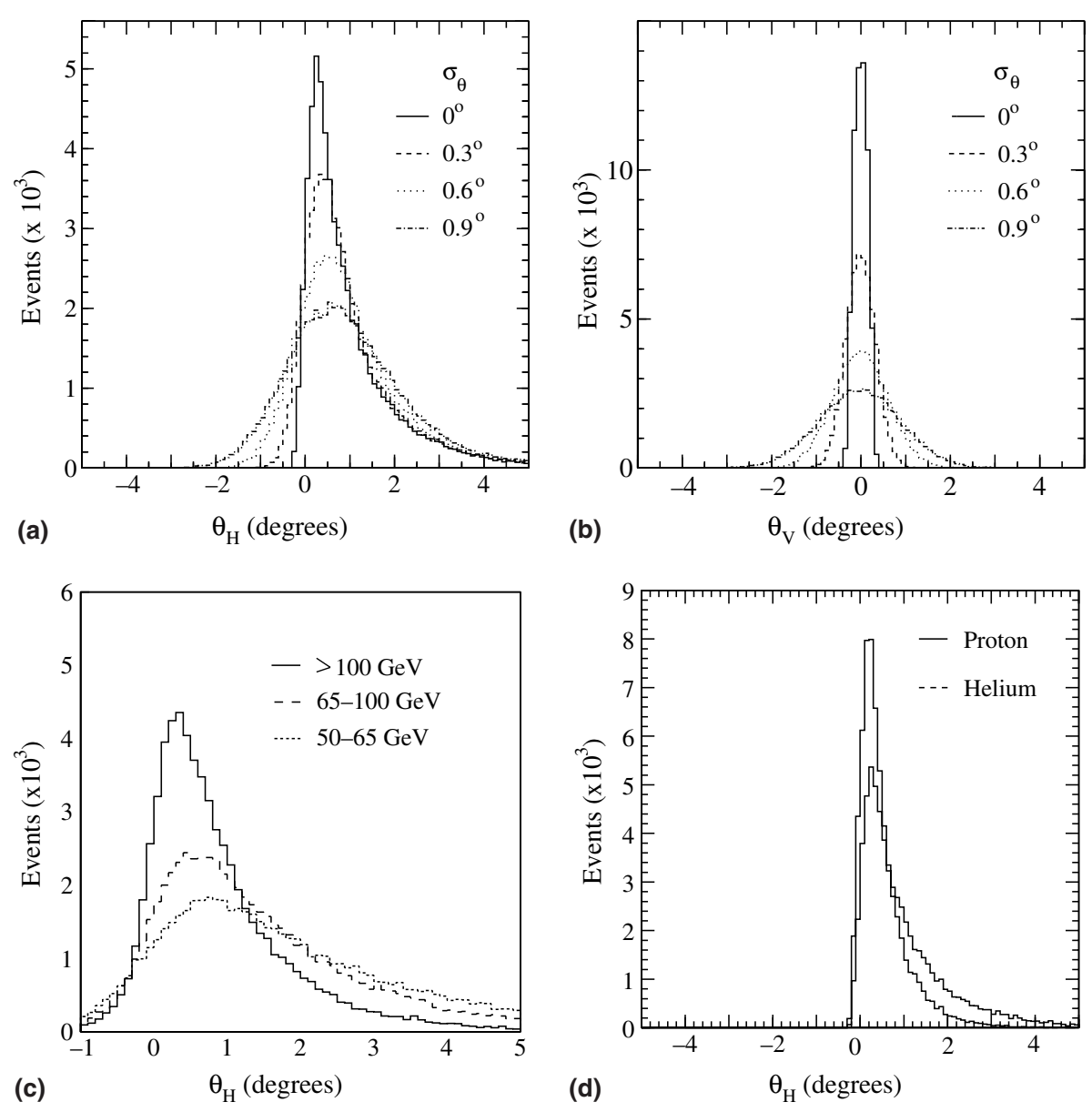

Fig. 6. Examples of results of the simulation of the shape of the Moon shadow: (a) $\theta_{\mathbf{H}}$ for $E_{\mu}>100 \mathrm{GeV}$ and for several hypothesis for the angular resolution, (b) $\theta_{\mathrm{V}}$ for $E_{\mu}>100 \mathrm{GeV}$ and for several hypothesis for the angular resolution, (c) $\theta_{\mathrm{H}}$ for three muon energy ranges, (d) $\theta_{\mathrm{H}}$ for proton and helium primaries for $E_{\mu}>100 \mathrm{GeV}$. All distributions are normalised to the same number of events. 

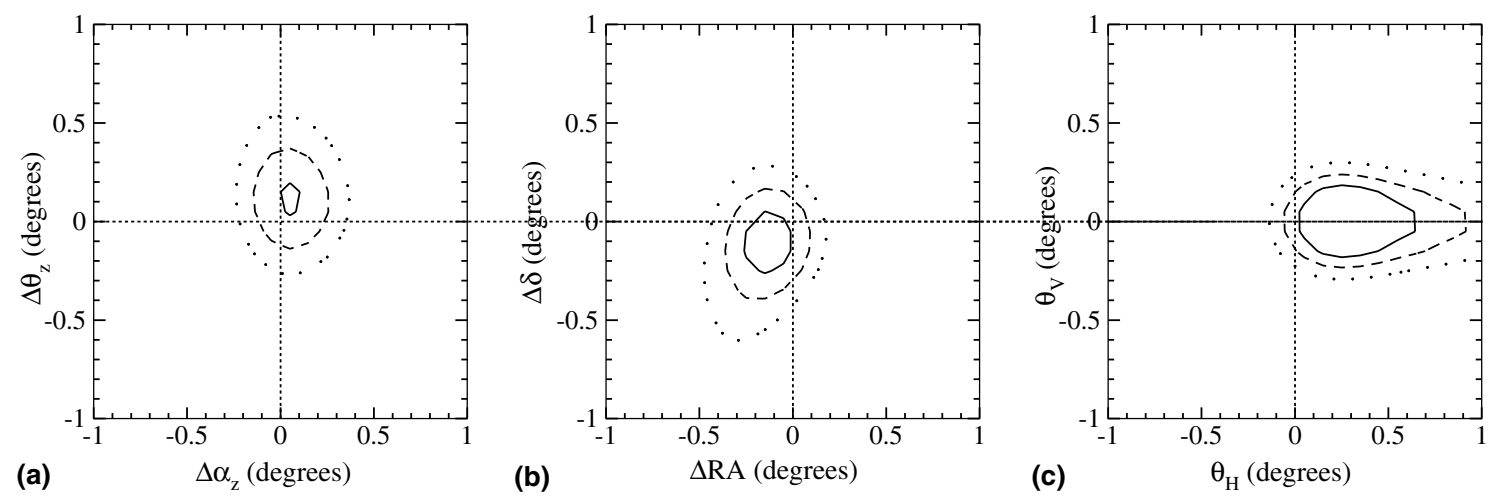

Fig. 7. Contour map of the simulated Moon shadow for $E_{\mu}>100 \mathrm{GeV}$ : (a) in the local coordinate system, (b) in the celestial coordinate system, (c) in the deflection system. For each system a total of $6 \cdot 10^{4}$ events have been simulated. The solid contour line defines the domain containing more than 700 events per sky unit cell (of size $0.1^{\circ} \times 0.1^{\circ}$ ), and the dashed and dotted lines lower minimal numbers. The deflection system obviously records the largest amount of cells containing at least 700 events each.

field are more visible in the deflection system. The search for a possible "anti-shadow" due to antiprotons is therefore performed in this last system.

There are two ways to use the simulation results in this analysis, either directly, or through a parametrisation. In the first case, simulated distributions represent the expectation values to be compared to the experimental data. In the second case, the adjustment of the parametric function to the data allows the extraction of the parameters. This is used to give a result on the observed effective angular resolution. The simulated shadow is parametrised as the product of two functions, one for each direction:

$f(x, y)=f_{\mathrm{H}}(x) \times f_{\mathrm{V}}(y)$,

$f_{\mathrm{V}}$ can be described as the projection on the vertical axis of the two-dimensional convolution of a disk (the Moon) with a Gaussian distribution, corresponding to an effective angular resolution for the muon momentum range considered:

$$
\begin{aligned}
f_{\mathrm{V}}(y)= & \int_{-R_{\text {Moon }}}^{+R_{\text {Moon }}} \frac{2}{\pi R_{\text {Moon }}^{2}} \sqrt{R_{\text {Moon }}^{2}-u^{2}} \\
& \times \frac{1}{\sigma \sqrt{2 \pi}} \mathrm{e}^{-\frac{(y-u)^{2}}{2 \sigma^{2}}} \mathrm{~d} u .
\end{aligned}
$$

For $f_{\mathrm{H}}$, no analytical description exists. However, it is found that a sum of two Landau distributions approximates reasonably well the shape in the direction parallel to the deflection:

$$
\begin{aligned}
f_{\mathrm{H}}(x)= & (1-c) \cdot L_{1}\left(x, a_{1}, b_{1}\right)+c \\
& \cdot L_{2}\left(x, a_{2}, b_{2}\right),
\end{aligned}
$$

with the parameters $a$ and $b$ acting respectively on the width and the position of the maximum of the distribution. For each selected value of the angular resolution, a number of events corresponding to 100 times the number of expected deficit events in the experiment is simulated. A good agreement between the simulated shape of the deficit and the parametrisation is observed.

\section{The Moon data-samples}

To define the muon direction in the sky, both local and terrestrial based coordinate systems are used. The Moon position is computed using the "SLALIB" library subroutines [35]. The error on the position calculation is estimated to be smaller than $0.01^{\circ}$, much smaller than the angular resolution or the Moon radius. The Moon is the nearest and the only astronomical object for which the position is significantly dependent on the observation location on Earth. This parallax effect is taken into account. It amounts to a few tenths of a degree.

Another effect is the change of the apparent size of the Moon as seen from the Earth due to the variation of the centre-to-centre distance from the Earth to the Moon between perigee and apogee. 
Calculations show that the Moon angular radius, as seen from the Earth, fluctuates between $0.25^{\circ}$ and $0.28^{\circ}$ during the whole data taking period.

The Moon declination is continuously changing inside a range of around $\pm 20^{\circ}$. In the local sky, the Moon follows a trajectory reaching a minimum zenith angle of $25^{\circ}$ for the experimental running period.

As the detector cannot be triggered above a certain zenith angle value, the Moon is only available for certain periods of time, each called a "cycle" in the following. Fig. 8 shows the Moon acceptance for the selected events for the two running years. Data were accumulated for five cycles in 1999 (73 transits) and nine cycles in 2000 (142 transits). The Moon was available for a total of $1557.5 \mathrm{~h}$.
The corresponding data-acquisition live-time is 1188.7 h $(76.3 \%)$.

\subsection{Data selection and monitoring}

A first selection isolates events coming from the direction of the Moon. For each event, the Moon position is computed in local coordinates and the space angle $\theta$ with the muon track direction is deduced. The track-reconstruction program requests at least one "triplet" (hits from three chambers in one of the octants) and one scintillator hit. Two hits in one octant (called a "doublet") are also allowed if a momentum measurement is possible, but two doublets are rejected. This constraint leads to the reconstruction of only one third of the total
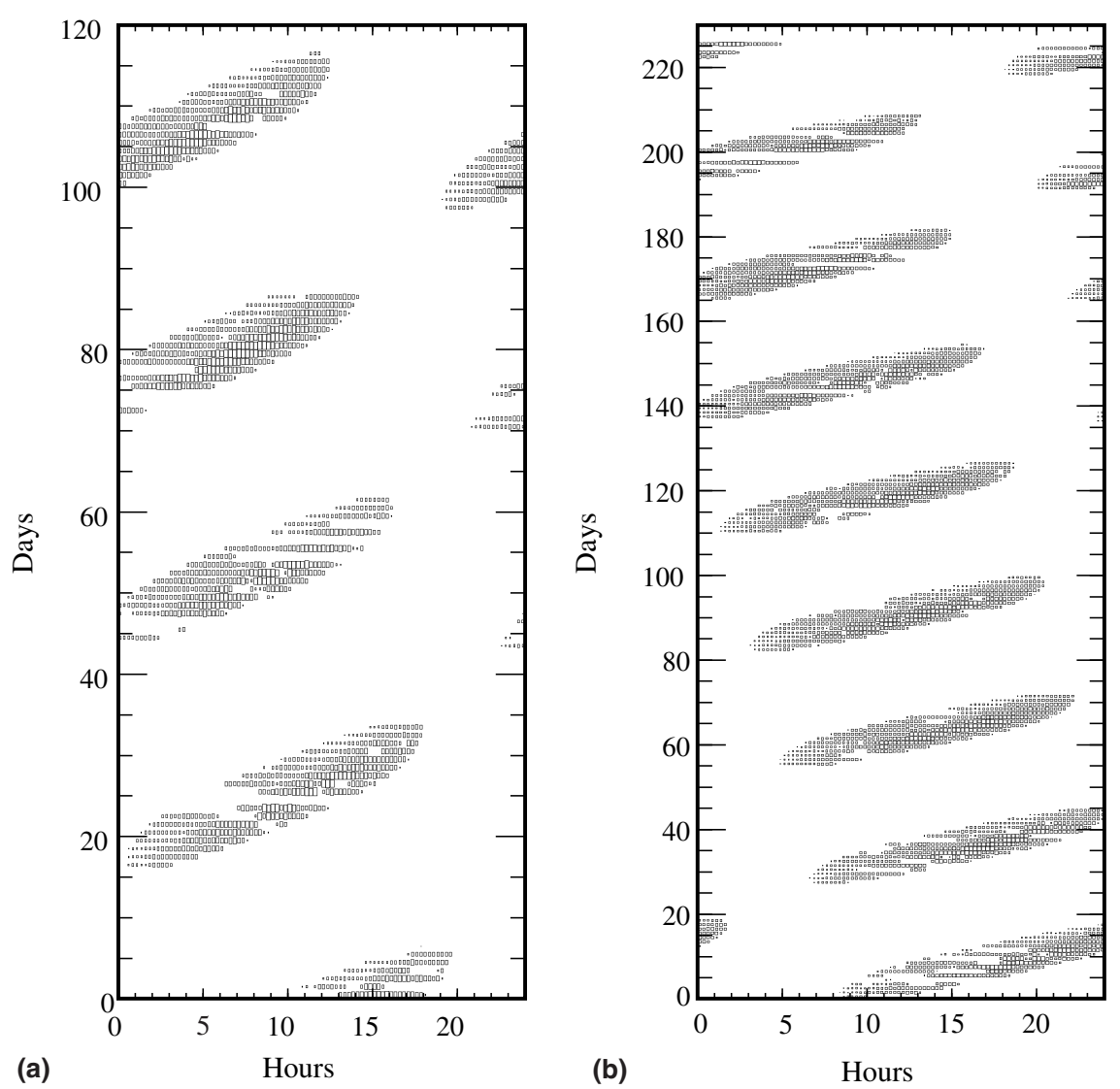

Fig. 8. Moon acceptance for (a) year 1999 and (b) year 2000. The plot shows the event arrival-time in days and hours. The running period includes 5 lunar cycles in 1999 and 9 in 2000. White bands during cycles correspond to periods in which either the detector or the data acquisition were not operational. 
number of events collected. The following cuts are applied at this level:

- Only one muon is reconstructed in each event.

- The angle with the Moon direction is less than $5^{\circ}$.

- The muon momentum is more than $50 \mathrm{GeV}$. This cut is motivated by the fact that lowenergy muons have little correlation with the direction of the primary cosmic ray.

- The Moon zenith angle is less than $60^{\circ}$. Above this value reconstruction becomes more difficult and the trigger efficiency is low.

- The backtracking of the measured muon track in the detector up to the surface is successful.

- Events with timing uncertainties, amounting to $0.2 \%$ of the total, are rejected as correct time information is needed to compute precisely the Moon position.

When these cuts are applied, a total of $6.71 \times 10^{5}$ events are selected, out of which $2.11 \times 10^{5}$ in 1999 and $4.60 \times 10^{5}$ in 2000 .

To monitor the data, some variables are carefully tested as a function of time. Among them are the number of selected events, the number of high-energy events, the $\mu^{+} / \mu^{-}$charge ratio, the proportion of muons including 2 subtracks and the proportion of high quality subtracks in events. Di-muon events are also used. They are taken from directions along the Moon trajectory using the same runs as in the Moon data. A total of more than 50000 events are collected in this way. The event selection requires that at least one of the muons has two subtracks and that the minimum muon momentum is $50 \mathrm{GeV}$. The di-muon space angle distribution is monitored.

Stability with time is an indication that no major problem perturbed the collection of data during all the running of the experiment. No major instability is observed for the whole running period.

\subsection{Background determination}

Searching for a point source (or shadow) entails the counting of the number of events in an angular bin containing the possible signal (signal bin) and comparing it to the number of background events expected in this bin. The background is a function $B\left(\theta_{z}, \alpha_{z}, t\right)$ of the zenith angle $\theta_{z}$, the azimuth angle $\alpha_{z}$, and the time $t$.

Apart from the $\theta_{z}$ dependence due to the changing thickness of the atmosphere, there is a strong spatial dependence of the reconstruction efficiency due to the arrangement of the muon chambers in octants and to the constraints imposed on the track reconstruction. The background is therefore evaluated by counting events in regions that were exposed for the same amounts of time to the same directions of the sky as the signal bin.

The global rate is changing with time, due to modifications in the detector hardware or in the detector environment (noise dependence, local atmospheric temperature and pressure dependence). A correction has therefore to be applied. In general time and spatial angular dependence are independent and the spatial acceptance is nearly constant. If not, one has to consider sufficiently small time slices so that this is valid.

The background is determined by measuring the number of events due to "fake Moons". These are bins which cross a given region in the sky either earlier or later than the signal bin in different runs. Averaging background samples on both sides of the signal bin removes effects of changes in the event rate which are linear in time. Four "fake Moons" are used, one hour and two hours before and after the real Moon position. When considering both running years, ten samples are available for the background evaluation (the two signal samples and eight background samples).

The projections on the azimuth and on the zenith axes are shown in Fig. 9a and b. In these examples, the merged data from all samples are used. The distributions are fitted by a straight line. The fitted slope parameters from the individual samples are shown in Fig. 9c and $\mathrm{d}$ for the azimuth and zenith directions respectively. There is only a slight positive variation of the rate as function of the azimuth angle and all the samples give statistically compatible results. The variation with the zenith angle is more important. The rate is decreasing for large zenith angles, a consequence of the acceptance. Moreover sample-to-sample fluctuations are larger.

Each sample is fitted by a plane in the azimuth-zenith coordinate system. The expected 

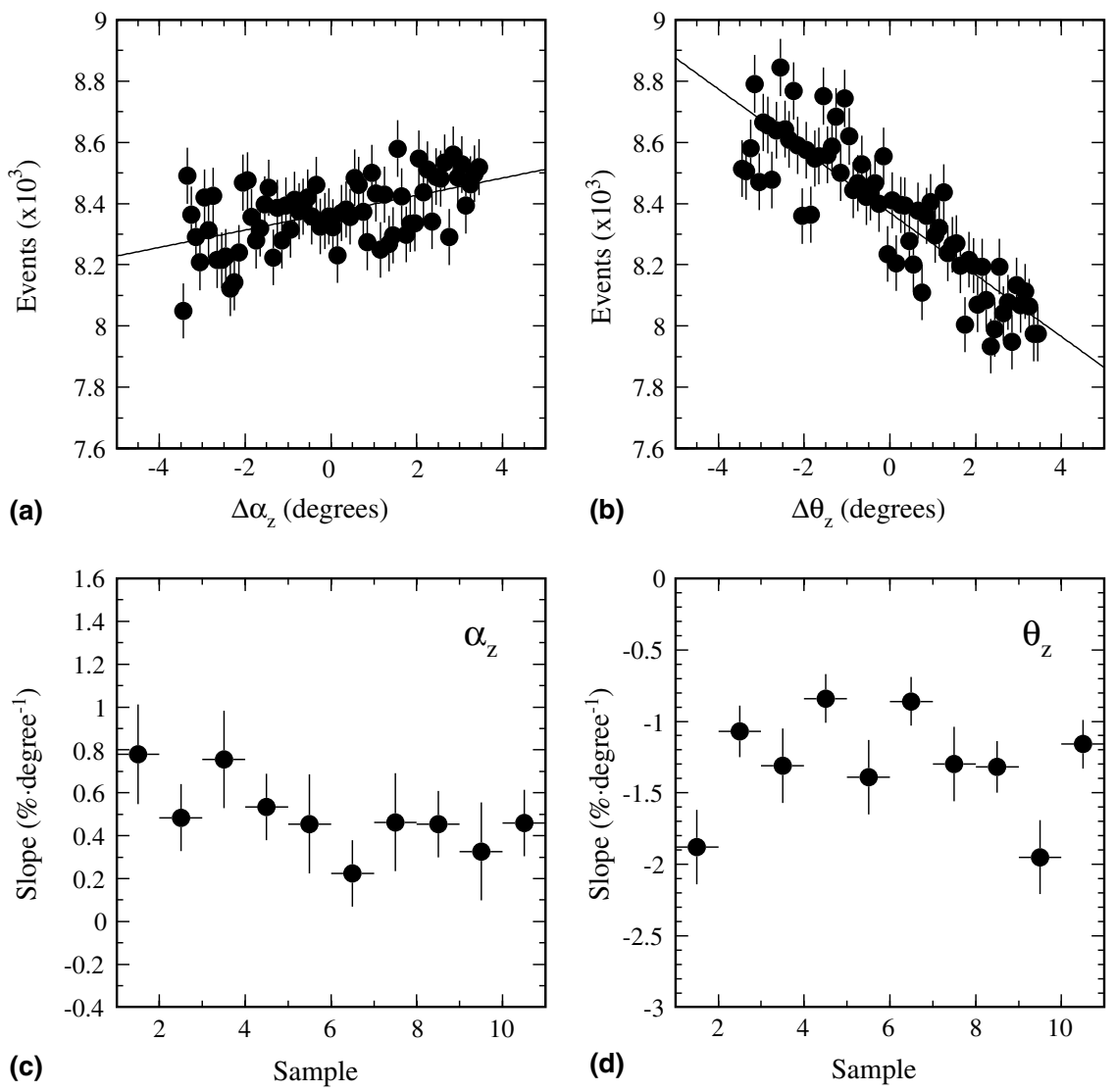

Fig. 9. Number of events versus (a) the azimuth and (b) the zenith direction, using the merged data from the 10 samples. The origin is taken at the real or "fake Moon" position. (c) Slope parameters extracted for the 10 considered samples in the azimuth direction and (d) in the zenith direction. The first 2 bins are from signal and the remaining from background.

background density at the origin (real or "fake Moon" position) is computed, expressed by the number of events in the Moon solid angle, $\Omega_{\text {Moon }}$, for the given live-time of the whole experiment. Fluctuations between samples are at the level of a few percent, much greater than the statistical uncertainties. Therefore systematic uncertainties due to live-time errors and acceptance or rate changes with time dominate. Following the hypothesis mentioned above that time and spatial angular dependence are independent, a further normalisation correction-factor based on the overall number of events for each sample can be applied. This is obtained from the total number of events inside an annulus around the nominal Moon position $\left(3^{\circ}<\theta<5^{\circ}\right)$. After this normalisation, differences are at the $0.1 \%$ level. When apply- ing the above procedure, there is no significant difference in the evaluation of the background using different samples. The systematic uncertainties in the knowledge of the background rate at the Moon position are negligible compared to the statistical uncertainties on the signal. The result averaged for the two years is $542 \pm 0.6$ events $/ \Omega_{\text {Moon }} \simeq 2366 \pm 3$ events $/ \mathrm{deg}^{2}$ for $E_{\mu}>$ $100 \mathrm{GeV}$ and $677 \pm 1.0$ events $/ \Omega_{\mathrm{Moon}} \simeq 2956 \pm 4$ events/deg ${ }^{2}$ for $65 \mathrm{GeV}<E_{\mu}<100 \mathrm{GeV}$.

\section{Event-deficit analysis}

In the local coordinate system, evidence for a cosmic ray deficit introduced by the Moon is observed using a single angular variable. The 

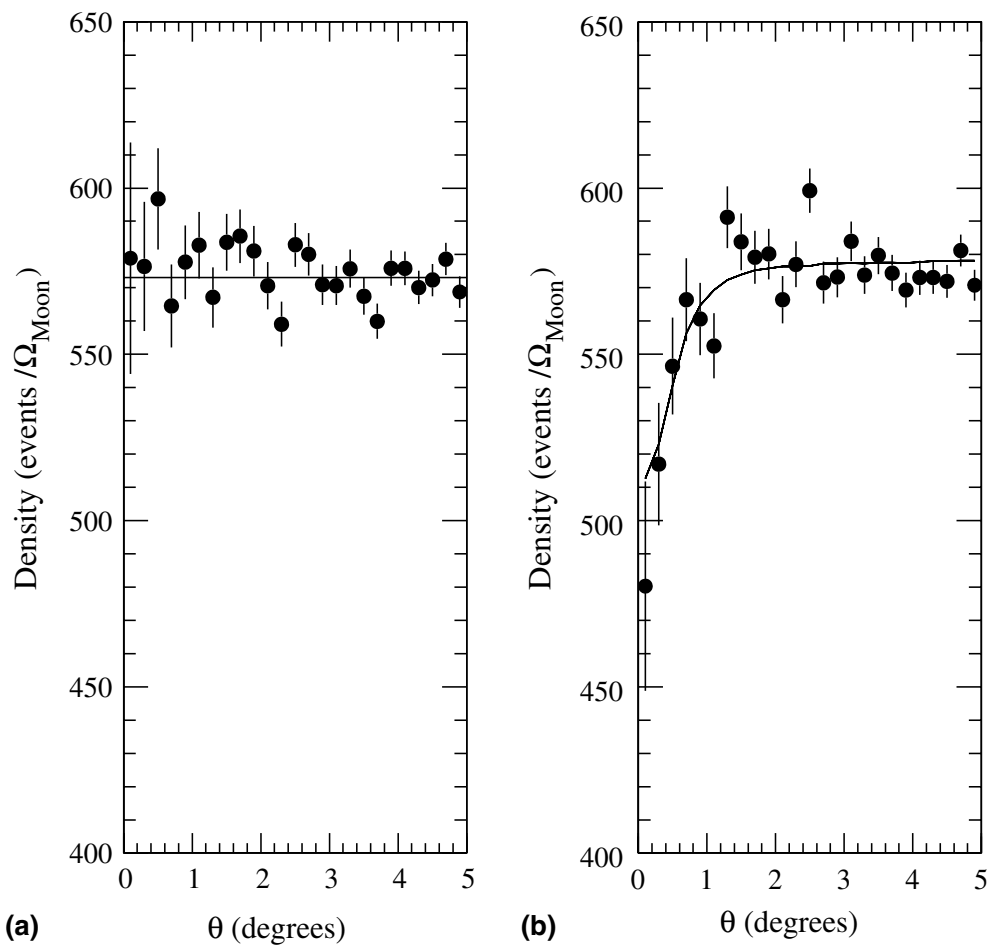

Fig. 10. Angular distributions for events/Moon-solid-angle with $E_{\mu}>100 \mathrm{GeV}$, (a) for a "fake Moon" shifted 1 hour behind its real position, (b) using the correct Moon position. Solid lines are the results of the simulation, including the angular resolution deduced from the study of di-muon events.

number of events shows a linear increase with increasing solid angle. Therefore the density of events is generally considered. In absence of any signal, plots must show a flat distribution. The plots of Fig. 10 show the results for $E_{\mu}>100 \mathrm{GeV}$ with a "fake Moon" shifted $1 \mathrm{~h}$ behind its real position along its trajectory (Fig. 10a) and with the Moon at its nominal position (Fig. 10b). In this last plot, a clear deficit of events in the first few bins is observed. This is attributed to the shadowing effect of the Moon.

Uncertainties in pointing and the influence of the geomagnetic field are also contributing to the shape of the deficit. The extraction of the experimental angular resolution and the measurement of the $\bar{p}$ content in primary cosmic rays are not possible on this one-dimensional distribution. In the following, a maximum likelihood method is used to disentangle the various factors. An interesting property of the "deflection coordinate system", which is based on the deflection induced by the Earth magnetic field, is to concentrate the Moon shadow deficit along one axis, thus optimising the signal density. Therefore the analysis will be performed in this coordinate system with the two projection angles $\theta_{\mathbf{H}}$ and $\theta_{\mathrm{V}}$. Studies to investigate the effect of the muon momentum threshold on the deficit lead to the definition of two samples, a "high energy (HE)" sample for $E_{\mu}>100 \mathrm{GeV}$ and a "low energy (LE)" sample for $65 \mathrm{GeV}<E_{\mu}<100 \mathrm{GeV}$.

Fig. 11 shows "shadow" plots concerning the data for both samples. Smoothing techniques are used. The way the background is computed is described later. For the analysis, "raw" spectra are used. A binning of $0.1^{\circ}$ is chosen in each direction.

The shape of the shadow is more elongated in the case of the LE sample and its position is shifted further. The position and the shape of the Moonrelated deficit mainly depend on the magnetic deflection undergone by the primary particle associ- 

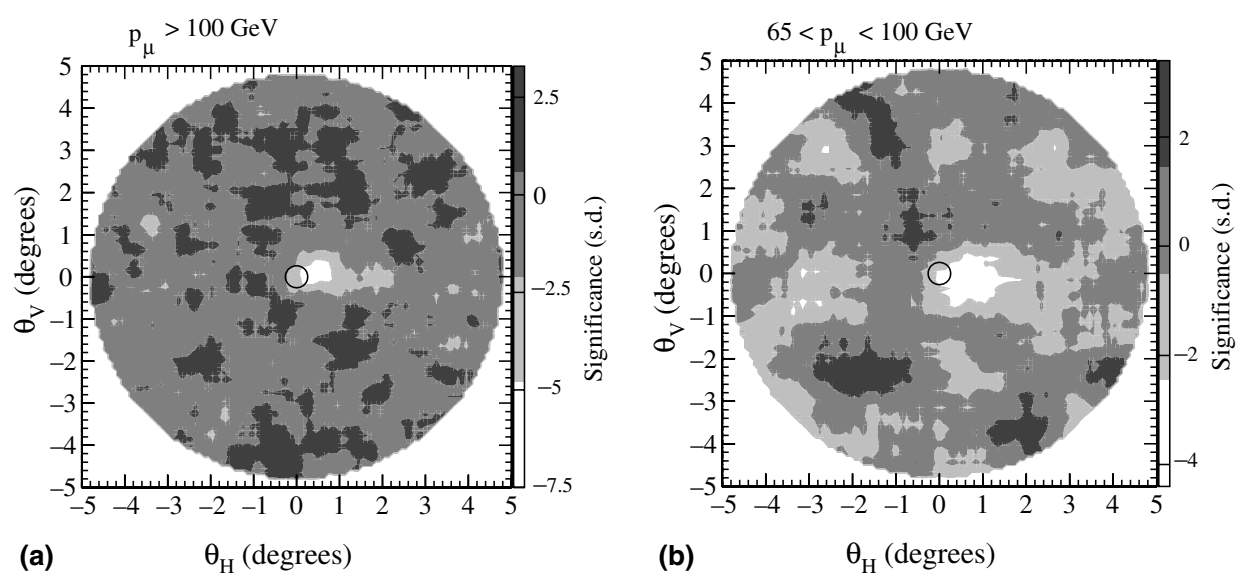

Fig. 11. Results obtained in the deflection system for: (a) the high-energy sample, (b) the low-energy sample. In both cases, smoothing techniques have been applied. A circle indicates the true position of the Moon. The vertical grey scale shows the significance in standard deviation units; negative values correspond to an event deficit.

ated with each muon of the sample and on the effective angular resolution $\sigma$. Both effects are taken into account in the simulation. The effective angular resolution includes the muon production-angle inside the air shower, the multiple scattering in the molasse above the detector and the intrinsic angular-resolution due to muon-chamber resolution, alignment and reconstruction. The smearing due to the multiple scattering is the main component of $\sigma$. A parametrisation of the simulation output for each value of $\sigma$ and for each sample was described in Section 3.5. It is used in the maximum likelihood fit to allow the extraction of $\sigma$ as a free parameter.

\subsection{Analysis procedure}

The probability to have $n_{i, j}$ events in bin $(i, j)$ of the $\theta_{\mathrm{V}}-\theta_{\mathrm{H}}$ distribution, when $g\left(x_{i}, y_{j}\right)$ events are expected is described by Poisson statistics. The logarithm of the likelihood function can be written as:

$$
\begin{aligned}
\ln \mathscr{L} \equiv & \sum_{i, j=1}^{N} \ln \left(P_{i, j}\right) \\
= & \sum_{i, j=1}^{N}\left[n_{i, j} \ln \left(g\left(x_{i}, y_{j}\right)\right)-g\left(x_{i}, y_{j}\right)\right. \\
& \left.\quad-\ln \left(n_{i, j} !\right)\right] .
\end{aligned}
$$

For practical reasons, the likelihood function is normalised and a minimum $\Delta \ln \mathscr{L}_{\mathrm{m}}$ is searched as a function of the parameter values. The twodimensional distributions are the result of the combination of three different components:

- a smooth background, which can be fitted with a plane,

- the proton and helium deficit introduced by the Moon shadow,

- the antiproton deficit if any.

The simulation provides a description of the proton deficit. A similar description is used for the antiproton deficit. However, in the deflection coordinate system, the shadow position is inverted with respect to the Moon centre. Also the shape of the antiproton shadow will differ from that of the protons, due to a possible different power index $\gamma$ of the energy spectrum. Thus the most general description of the data is

$$
\begin{aligned}
g(x, y)= & \underbrace{u_{x} x+u_{y} y+u_{z}}_{\text {background }}-\frac{N_{\text {miss }}}{1+r}[0.75 \\
& \times \underbrace{f_{1}\left(x-x_{0}, y-y_{0}, \sigma\right)}_{\text {p deficit }}+0.25 \\
& \times \underbrace{f_{2}\left(x-x_{0}, y-y_{0}, \sigma\right)}_{\text {He deficit }}+r \\
& \times \underbrace{f_{3}\left(x_{0, \overline{\mathrm{p}}}-x, y_{0, \overline{\mathrm{p}}}-y, \sigma_{\overline{\mathrm{p}})}\right)}_{\overline{\mathrm{p}} \text { deficit }},
\end{aligned}
$$


where $f_{1}, f_{2}$ and $f_{3}$ are the shadow functions defined by Eq. (7), respectively for protons, helium nuclei and antiprotons. The parameters that can vary for the maximum likelihood fit are the angular resolutions $\sigma$, the positions of the deficits $\left(x_{0}, y_{0}\right)$, the number of missing events $N_{\text {miss }}$ and $r$, the $\overline{\mathrm{p}}$ content. The parameters $u_{x}, u_{y}, u_{z}$ describe the background.

The influence of different power indexes $\gamma(-1.7$ to -3.7$)$ of the antiproton energy spectrum on the final limit of the antiproton to proton ratio presented in this paper is studied, as well as a re-analysis of the data assuming also different values of $\gamma$. Variations of less than $20 \%$ with respect to a simplified model, where the power index is assumed identical for protons and antiprotons are obtained. The interpretation of this conclusion may be explained by the fact that for a steep antiproton spectrum the deflection of most antiprotons is relatively large, but the muon statistics small, due to the lower average primary energy. In the case of a flatter spectrum the deviation is small, but the muon statistics larger. The two effects cancel each other, providing a negligible influence on the limit of the $\bar{p} / p$ ratio. For simplicity we present only the result of the analysis with the assumption of equal power indexes, reducing thus the number of free fit parameters to eight $\left(f_{3}=f_{1}, x_{0, \overline{\mathrm{p}}}=-x_{0}, y_{0, \overline{\mathrm{p}}},=\right.$ $\left.-y_{0}, \sigma_{\overline{\mathrm{p}}}=\sigma\right)$.

Instead of trying to extract directly the eight parameters with the simplified equation (11), the analysis proceeds in several steps.

\subsection{Background estimation}

A first determination of the background parameters is performed using the ring-data defined as $3^{\circ}<\theta<5^{\circ}$, where $\theta$ is the angle between the muon and the nominal position of the Moon. The cut excludes the cells in the proton and antiproton deficit regions. The event density at the nominal Moon position is known at the $0.3 \%$ level. This uncertainty corresponds to the statistics used for its determination.

No significant changes in the parameter values are found when Eq. (11) is applied to the whole angular range and all the parameters are considered free.

\subsection{The Moon-shadow analysis}

In this step no antiprotons are supposed in the primary flux. The effective angular resolution of the detector, together with the pointing uncertainties, are obtained from the observation of the matter deficit only. Results of the maximum likelihood fit for the two samples are given in Table 1. As an example, two-dimensional $68 \%$ and $90 \%$ confidence level contour curves for the parameters $N_{\text {miss }}$ and $\sigma$ are shown in Fig. 12a for the case of the HE sample.

Pointing errors are given by horizontal or vertical offsets between data and simulation in the determination of the deficit position $x_{0}$ and $y_{0}$. Both values are small $\left(\leqslant 0.1^{\circ}\right)$.

Values related to the absolute position, $x_{0}$, and to the extension of the deficit (FWHM, full width at half maximum) in the horizontal direction $\theta_{\mathrm{H}}$ show clearly a momentum dependence. In the $\theta_{\mathrm{V}}$ direction, no shift is observed and the width is mainly the result of the effect of $\sigma$.

Values of $N_{\text {miss }}$ are extracted from the fit. However $N_{\text {miss }}$ can be also directly deduced from $\Phi$, the flux measurement around the Moon direction, $N_{\text {miss }}=\Phi \times T_{\text {live }} \times \Omega_{\text {Moon }}$ where $T_{\text {live }}$ is the livetime corresponding to the Moon observation.

Table 1

Results obtained in the fit of the matter deficit

\begin{tabular}{lllll}
\hline Parameter & HE measured & HE expected & LE measured & LE expected \\
\hline$x_{0}$ & $0.33 \pm 0.08$ & 0.26 & $0.53 \pm 0.13$ & 0.48 \\
$y_{0}$ FWHM & $0.05 \pm 0.05$ & 0.0 & $-0.10 \pm 0.08$ & 0.0 \\
$N_{\text {miss }}$ & $1.07_{-0.04}^{+0.07}$ & 1.03 & $1.80 \pm 0.15$ & 1.87 \\
Significance & $575_{-87}^{+97}$ & $546 \pm 5$ & $536_{-127}^{+133}$ & $683 \pm 6$ \\
\hline
\end{tabular}



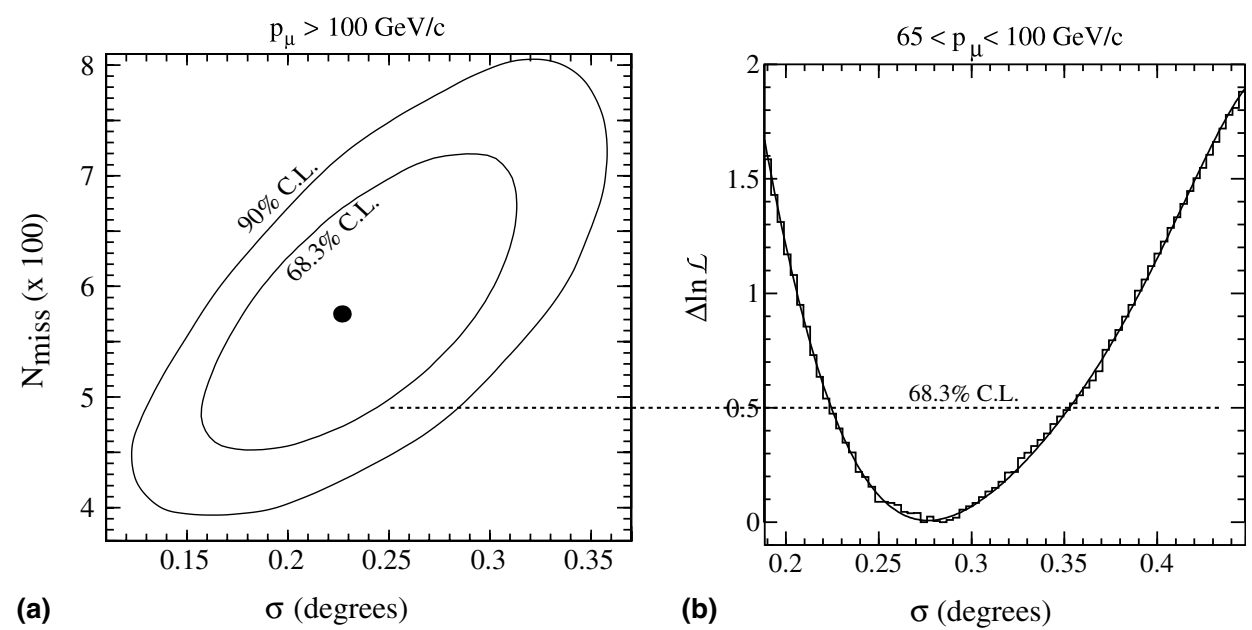

Fig. 12. (a) Two-dimensional $68 \%$ and $90 \%$ C.L. contour curves for the parameters $N_{\text {miss }}$ and $\sigma$ for the HE sample, (b) $\Delta \ln \mathscr{L}$ versus $\sigma$ for the LE sample. $N_{\text {miss }}$ has been constrained. The dashed line for $\Delta \ln \mathscr{L}=0.5$ is used to determine the $68.3 \%$ central confidence interval.

The main contribution to the uncertainty is related to the Moon solid angle. At any time the precision of the calculations of the Moon radius $R_{\mathrm{M}}$, from the SLALIB subroutines [35], is estimated to be $0.4 \%$ and the time distribution of the Moon events is very well known. The uncertainty on the solid angle, proportional to $R_{\mathrm{M}}^{2}$ is thus $0.8 \%$. The flux is known at the level of $0.3 \%$ and the uncertainty on the live-time is still smaller. In total, the precision on the most probable value of $N_{\text {miss }}$ is estimated to be around $1 \%$. The expected values of $N_{\text {miss }}$ are shown in Table 1. This knowledge of $N_{\text {miss }}$ is introduced in the likelihood function and allows an improvement in the determination of the remaining parameter $\sigma . N_{\text {miss }}$ is constrained to its most probable value, with a $1 \%$ uncertainty. The results are illustrated in Fig. 12b for the LE sample with the plot of $\Delta \ln \mathscr{L}$ versus $\sigma$. The experimental results are $\sigma=(0.22 \pm 0.04)^{\circ}$ for the HE sample and $\sigma=\left(0.28_{-0.05}^{+0.08}\right)^{\circ}$ for the LE sample. These numbers refer to an effective angular resolution valid for the set of selected events, integrated over the momentum distribution of the data and the directional range of the Moon events. For the HE sample, Fig. 13 shows a comparison of a projected band of data around the nominal position of the Moon with the fitted results corresponding to Table 1 .
As a cross check, the effective angular resolution is also obtained by a fit to the one-dimensional deficit distribution. The values discussed above are confirmed, albeit with much larger uncertainties.

\subsection{The antiproton search}

To set a limit on a possible cosmic ray antiproton component, the number of missing events $N_{\text {miss }}$ is supposed to be shared between protons, helium and antiprotons. The total number of missing events has been constrained to the expected value. The antiproton deficit is described like the proton deficit with the corresponding parametrisation function symmetric to the proton one with respect to the Moon position.

As mentioned in Section 3.1.2, the value of $\sigma$ can be deduced from the simulation. Comparison with data in di-muon events shows a good agreement. Contrary to the situation in the Moon-shadow experiment, high statistics is available and detailed investigations are possible. The obtained results are $\sigma=(0.24 \pm 0.01)^{\circ}$ for the HE sample and $\sigma=(0.38 \pm 0.02)^{\circ}$ for the LE sample. Uncertainties are better than those obtained above. This angular information is used and a maximum likelihood fit is performed using the $\bar{p}$ content as a free parameter. 


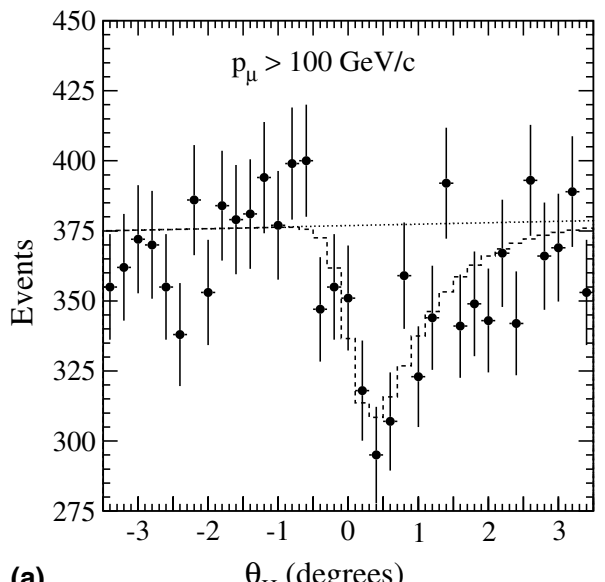

(a)

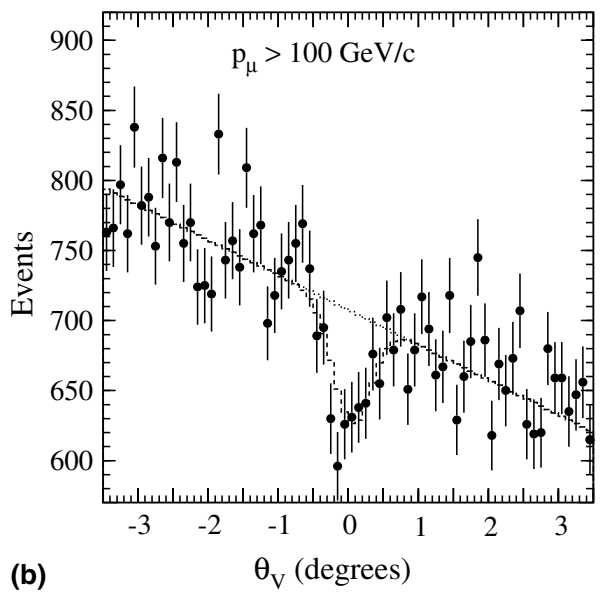

Fig. 13. Comparison between data and fitted results for the HE sample in a projection on (a) the horizontal axis, (b) the vertical axis. Differences in the projected bandwidths (a: 0.8 degrees, b: 3.0 degrees) explain the differences of the observed number of events. The dotted lines represent the fitted average muon event number outside of the Moon region, and the dashed lines the fit to the data in the Moon region.

The HE and LE results are combined to give the final measurement. This is done simply by adding the likelihood logarithmic-functions of each range. The total significance of the deficit is 9.4 s.d. The uncertainty range is obtained by finding the points for which $\Delta \ln \mathscr{L}=\Delta \ln \mathscr{L}_{\mathrm{m}}+0.5$. The result is shown in Fig. 14 and one finds $r=\phi_{\overline{\mathrm{p}}} / \phi_{\text {matter }}=$ $-0.07 \pm 0.09$. The result is below a physical boundary (the $\overline{\mathrm{p}}$ content must be positive). An upper limit of 0.08 with $90 \%$ confidence level is

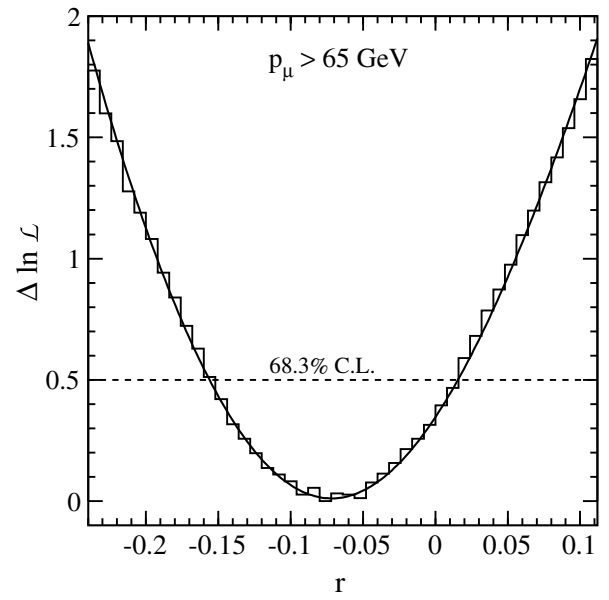

Fig. 14. $\Delta \ln \mathscr{L}$ as a function of $r$ (the $\overline{\mathrm{p}}$ content) for the whole data. The dashed line $\Delta \ln \mathscr{L}=0.5$ is used to determine the $68.3 \%$ central confidence interval.

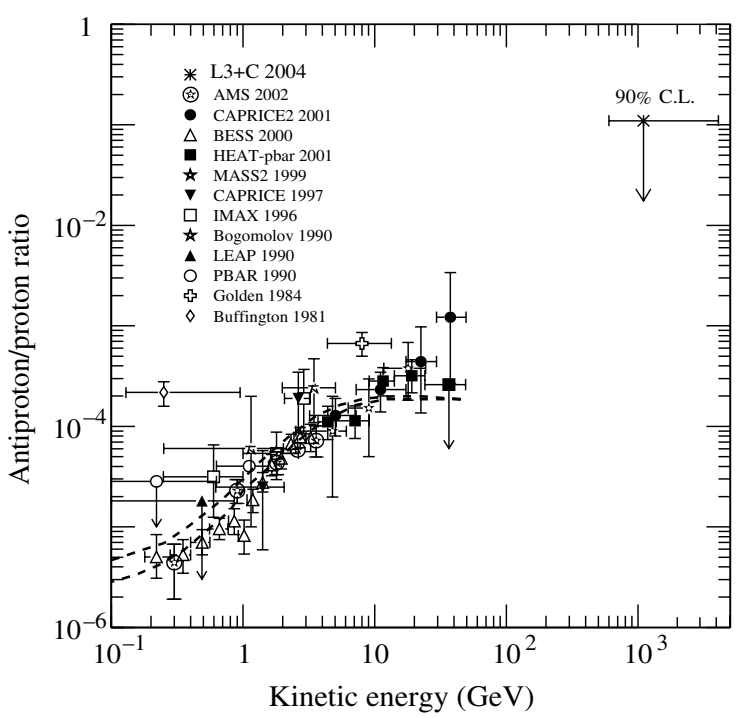

Fig. 15. Measurements of the ratio of the antiproton and proton fluxes versus the primary energy, including the L3 + C limit around $1 \mathrm{TeV}$. (The dashed lines show the range of the theoretical expectations [9].)

set using the unified approach [36]. With the assumed flux composition around $1 \mathrm{TeV}$ of $75 \%$ protons and $25 \%$ heavier nuclei responsible of the observed deficit, this corresponds to a $\overline{\mathrm{p}} / \mathrm{p}$ ratio 
of $r_{\overline{\mathrm{p}} / \mathrm{p}}=0.11$. Fig. 15 shows the L3 $+\mathrm{C}$ result together with other published values.

\section{Conclusions}

The L3 detector has collected more than $10^{10}$ triggers of cosmic ray muons during the years 1999 and 2000 in parallel with high-energy particle physics studies at the LEP accelerator at CERN. About $6.7 \times 10^{5}$ events, with a direction pointing to a $5.0^{\circ}$ cone around the Moon, are used and a Moon-shadow effect in cosmic rays is observed.

A two-dimensional analysis confirms that the alignment of the detector is correct to better than $0.2^{\circ}$ and that the size and the shape of the deficit are compatible with the expectations. Two sets of data corresponding to high $\left(E_{\mu}>100 \mathrm{GeV}\right)$ and low-energy muons $\left(65 \mathrm{GeV}<E_{\mu}<100 \mathrm{GeV}\right)$ lead to values of the effective angular resolution respectively of $(0.22 \pm 0.04)^{\circ}$ and $\left(0.28_{-0.05}^{+0.08}\right)^{\circ}$. These numbers include all effects due to the showering of the primary cosmic ray in the atmosphere, the multiple scattering in the molasse and the detector resolution. They describe correctly the observed event deficit. The observed significance of the Moon-shadow effect is 9.4 s.d. A significant effect due to the Earth magnetic field is observed. This is better seen in a coordinate system with axis respectively parallel and orthogonal to the deflection defined for each direction in the local sky. The offset and the extension of the shadow are clearly dependent on the muon momentum range considered. With the hypothesis that the presence of antiprotons in cosmic rays would lead to a symmetric shadow to the one due to protons, a measurement of the $\overline{\mathrm{p}}$ content is extracted from the data and is found to be $r=-0.07 \pm 0.09$. A $90 \%$ confidence level of 0.08 is set on $r$, corresponding to an antiproton over proton ratio of $r_{\overline{\mathrm{p}} / \mathrm{p}}=0.11$.

\section{Acknowledgements}

We wish to acknowledge the contributions of all the engineers and technicians who have participated in the construction and maintenance of the
L3 and L3 + C experiments. Those of us who are not from member states thank CERN for its hospitality and help.

\section{References}

[1] G.W. Clark, Phys. Rev. 108 (1957) 450.

[2] D.E. Alexandreas et al. CYGNUS Collaboration, Phys. Rev. D 43 (1991) 1735.

[3] A. Borione et al. CASA Collaboration, Phys. Rev. D 49 (1994) 1171.

[4] M. Amenomori et al. TIBET Collaboration, Phys. Rev. D 47 (1993) 2675.

[5] M. Ambrosio et al. MACRO Collaboration, Phys. Rev. D 59 (1999) 012003.

[6] M. Ambrosio et al. MACRO Collaboration, Astropart. Phys. 20 (2003) 145.

[7] J.H. Cobb et al. SOUDAN 2 Collaboration, Phys. Rev. D 61 (2000) 92002.

[8] M. Urban et al., Nucl. Phys. B (Proc. Suppl.) 14B (1990) 223.

[9] I.V. Moskalenko, Astrophys. J. 565 (2002) 280.

[10] M. Boezio et al. CAPRICE Collaboration, Astrophys. J. 561 (2001) 787.

[11] A.S. Beach et al. HEAT Collaboration, Phys. Rev. Lett. 87 (2001) 271101.

[12] L3 + C collaboration, J.-F. Parriaud, in Proceedings of the XIVth Rencontres de Blois, 2002, Blois, France;

L3 + C collaboration, P. Le Coultre, in Proceedings of the XIIth ISVHECRI, 2002, CERN, Geneva, Switzerland;

L3 + C collaboration, P. Le Coultre, in Proceedings of the IVth NOW, 2003, Kanasawa, Japan;

L3 + C collaboration, Y.P. Xu, in HE 3.3.1., Proceedings of the XXVIIIth ICRC, 2003, Tsukuba, Japan.

[13] TibetAS $\gamma$-collaboration, T. Kido, in HE 3.3.2., Proceedings of the XXVIIIth ICRC, 2003, Tsukuba, Japan.

[14] S.A. Stephens, Astron. and Astrophys. 149 (1985) 1.

[15] L3 + C collaboration, M. Unger, in Proceedings of the IXXth ECRS 2004, Florence, Italy.

[16] P. Achard et al. L3 Collaboration, Phys. Lett. B 598 (2004) 15.

[17] A. Barrau et al., Astron. and Astroph. 388 (2002) 676.

[18] L. Bergström, J. Edsjö, P. Ullio, Astrophys. J. 526 (1999) 215.

[19] P. Ullio, Preprint, astro-ph/9904086, 1999.

[20] F.W. Stecker and A.W. Wolfendale, Proceedings of the 19th ICRC, La Jolla, USA, 1985.

[21] D. Pomarède et al., Astropart. Phys. 14 (2001) 287.

[22] R. Atkins et al. MILAGRO Collaboration, Nucl. Instr. and Meth. A 449 (2000) 478.

[23] M.O. Wascko, PhD thesis, University of California, Riverside, 2001.

[24] F. Samuelson, Proceedings of the 27th ICRC, Hamburg, Germany, 2001. 
[25] B. Adeva et al. L3 Collaboration, Nucl. Instr. and Meth. A 289 (1990) 35.

[26] O. Adriani et al. L3 + C Collaboration, Nucl. Instr. and Meth. A 488 (2002) 209.

[27] J.-F. Parriaud, PhD thesis, Université Claude Bernard Lyon I, Lyon, 2003.

[28] R. Brun et al., GEANT Version 3.15, preprint DD/EE/84-1 (1984), revised 1987.

[29] D. Heck et al., CORSIKA, Version 6.019, Report FZKA 6019, 1998.

[30] IGRF: International Association of Geomagnetism and Astronomy (IAGA); DGRF95.DAT and IGRF00.DAT for 1999 data, and IGRF00.DAT and IGRF00S.DAT for 2000 data.
[31] B. Wiebel-Sooth et al., Astron. and Astroph. 330 (1998) 389.

[32] A.V. Apanasenko et al. RUNJOB Collaboration, Astropart. Phys. 16 (2001) 13.

[33] M. Aguilar et al. AMS Collaboration, Phys. Rep. 366 (2002) 331; Phys. Lett. B 490 (2000) 27.

[34] S. Haino et al. BESS collaboration, Phys. Lett. B 594 (2004) 35.

[35] SLALIB - A positional Astronomy Library, Rutherford Appleton Laboratory. Available from: http://www.eso.org/ science/scisoft/star/sun67.htx/sun67.html.

[36] G. Feldman, R. Cousins, Phys. Rev. D 57 (1998) 3873. 